\title{
Us and Them (and It): Social Orientation, Privacy Concerns, and Expected Use of Pandemic-Tracking Apps in the United States
}

\author{
John S. Seberger \\ Indiana University Bloomington \\ Bloomington, Indiana \\ jseberge@indiana.edu
}

\author{
Sameer Patil \\ Indiana University Bloomington \\ Bloomington, Indiana \\ patil@indiana.edu
}

\begin{abstract}
The deployment of technologies to track and mitigate the spread COVID-19 has surfaced tensions between individual autonomy and the collective good. These tensions reflect a conflict between two central concerns: (i) effectively controlling the spread of the pandemic and (ii) respecting individual rights, values, and freedoms. We explored these tensions in an online experiment $(n=389)$ designed to identify the influence of social orientation and communicative framing on perceptions and expected use of pandemic-tracking apps. We found that social orientation is a statistically significant predictor of app perception and expected use, with collectivist social orientation associated with higher levels and individualist social orientation with lower levels for both aspects. We found interactions between social orientation and communicative framing, as well as a connection between privacy concerns and expected duration of app use. Our findings hold important implications for the design, deployment, and adoption of technology for the public good. Shaping the post-pandemic social contract requires considering the long-term sociocultural impact of these technological solutions.
\end{abstract}

\section{CCS CONCEPTS}

- Human-centered computing $\rightarrow$ Empirical studies in $\mathrm{HCI}$; Empirical studies in collaborative and social computing; Empirical studies in ubiquitous and mobile computing; • Security and privacy $\rightarrow$ Social aspects of security and privacy.

\section{KEYWORDS}

COVID-19, pandemic, privacy, social orientation, IND-COL, social framing, social contract, contact tracing, surveillance, individualism, collectivism, smartphone apps, mobile apps, MUIPC

\section{ACM Reference Format:}

John S. Seberger and Sameer Patil. 2021. Us and Them (and It): Social Orientation, Privacy Concerns, and Expected Use of Pandemic-Tracking Apps in the United States. In CHI Conference on Human Factors in Computing Systems (CHI '21), May 8-13, 2021, Yokohama, fapan. ACM, New York, NY, USA, 19 pages. https://doi.org/10.1145/3411764.3445485

This work is licensed under a Creative Commons Attribution-NonCommercial International 4.0 License.

CHI '21, May 8-13, 2021, Yokohama, Japan

(c) 2021 Copyright held by the owner/author(s).

ACM ISBN 978-1-4503-8096-6/21/05.

https://doi.org/10.1145/3411764.3445485

\section{INTRODUCTION}

The ongoing novel coronavirus pandemic (COVID-19) has greatly affected daily life the world over. Just as COVID-19 affects our present, our responses to it will affect our collective futures up to, and including, the economic and philosophical modes of production that constitute modernity [43]. Various stakeholders, such as clinicians, epidemiologists, technologists, and governments, have supported the development of digitally augmented approaches to slow the spread and limit the impact of COVID-19. At the same time, society must not lose sight of the larger issues that emerge from the meeting of the pandemic and techno-solutionism. These include, but are not limited to, ethical quagmires [38], the furtherance of surveillance culture $[15,30,84]$, and the potential proliferation of user-centered privacy concerns $[19,39,52]$. We contend that the immediate medical and technological concerns raised by the COVID-19 pandemic must be contextualized by the broader system into which pandemic-related technology will be deployed: the social contract [60].

The social contract generically refers to the formalized system of power relationships between governments, institutions, and citizens. These power relationships are responsible for conferring legitimacy to governments and institutions [60]. Thus, these relationships are integral to the development of people's expectations of everyday life. The sociotechnical issues raised by the COVID-19 pandemic imply a potential social contract that foregrounds the role of technology $[13,60]$, possibly undermining the collective good of the population. Therefore, we must understand the potential interaction between techno-solutionism and the emergent social contract even as we develop and adopt the technologies we deem necessary to combat the pandemic. As the Senior Digital Rights Researcher at Human Rights Watch, Deborah Brown, has said, "Time is of the essence, but the pandemic is no time to move fast and break things" [35]. The measures we take today in the name of survival will become the infrastructural supports of the post-pandemic social contract, healthy or not. To understand the characteristics of the potential post-pandemic social contract, it is necessary to investigate its antecedents through the human-centered, sociotechnical lens of values, such as privacy.

Much ongoing work at the intersection of technology and pandemic response is aimed at understanding user perceptions and privacy concerns regarding pandemic-tracking apps (e.g., [8, 19, 31, $32,57,81]$ ). We contend that pandemic-tracking apps represent a novel context for mobile health (mHealth) in which mobile technologies are integrally entwined in the discourse of a born-digital infection, one which should be explored separately from, but in tandem with, canonical work at the intersection of usable privacy and mHealth. 
We begin with the assumption that usable privacy is an appropriate analytical lens, but not the only one. Effective responses to the pandemic require individual actions for the collective good as well as interaction and cooperation at the community and societal levels. Nuanced and contextual relationships between the individual, community, and society constitute the theater of privacy. These relationships, however, also serve a greater function. At a basic level, they are simultaneously background and performance of the social contract. It is therefore essential to understand how concepts of self in relation to social surroundings-social orientation-might affect the perceptions and adoption of pandemic-tracking apps. However, no efforts to-date have examined people's perceptions of pandemictracking technologies through the lens of social orientation.

To fill this gap, we examined the influence of social orientation, social framing, and utility framing on people's perceptions and expected use of pandemic-tracking apps within the United States. We similarly investigated the relationships between perceptions and expected use of pandemic-tracking apps and mobile user privacy concerns. Broadly, social orientation refers to the way in which people understand themselves in relation to their social surroundings. The most well-known social orientation categories are individualist and collectivist [70]. Framing refers to the language that describes a concept or an artifact [74]. Specifically, we consider social framing as the characterization of an app in individualist or collectivist terms. For example, using phrases such as "you personally" invokes an individualist social framing. Similarly, we define utility framing in terms of descriptions of outcomes derived from potential use of an app. For instance, "a 14-day quarantine and health monitoring" is an intrusive outcome.

We designed our study to answer four research questions:

RQ1: Does social orientation predict the perception and expected use of pandemic-tracking apps?

RQ2: Does the social framing used to describe pandemic-tracking apps predict the perception and expected use?

RQ3: Does the app's utility framing predict the perception and expected use of pandemic-tracking apps?

RQ4: Do mobile privacy concerns relate to the perception and expected use of pandemic-tracking apps?

To answer these questions, we conducted an online experiment between April 22 and May 22, 2020 via the Amazon Mechanical Turk (AMT) platform $(n=389)$. Our experiment examined the predictive value of social orientation, social framing, and utility framing on people's perceptions, expected honest reporting of symptoms, expectation to take app-recommended infection-prevention measures, and expected continued post-pandemic use of pandemic-tracking apps for general health monitoring. In addition, we investigated whether the relationships between these constructs are affected by privacy concerns and demographics.

We found perceived benefit and expected use of pandemic-tracking apps to be associated with social orientation and social framing, but not utility framing. While social framing is associated with expected continued use after the pandemic, it does not impact other facets of expected use. We further found that privacy concerns are unrelated to perceived benefit of pandemic-tracking apps, but exhibit a statistically significant negative correlation with expected post-pandemic use.
Based on the findings, we make the following contributions:

- We demonstrate the relationship between social orientation and perceived benefit and expected use of pandemic-tracking apps;

- We identify an unexpected relationship between collective social orientation and individualist social framing that needs to be considered in the design and deployment of pandemicrelated solutions involving the use of technology;

- We identify a need for presenting technologies that may involve trade-offs between individual needs and the collective good by via communication tailored to individualists.

In the sections that follow, we situate our work in the literature of psychology, mobile privacy, and mHealth. We use this literature to derive specific hypotheses related to each of our research questions. Next, we describe how we operationalized the relevant constructs with an instrument that we used to conduct an online experiment. We proceed to present the results of our analyses and discuss the findings pertaining to each hypothesis. We then explain a few limitations and provide directions for future work. We conclude by emphasizing that the post-pandemic social contract will be influenced by the complex relationship between immediate public health needs, long term surveillance concerns, and people's social orientations.

\section{RELATED WORK}

The literature related to our research covers concepts from psychology and considerations from mHealth and usable privacy. In this section, we review and synthesize salient works from these areas and use them to derive our hypotheses.

\subsection{Social Orientation}

Broadly, social orientation refers to the means by which the self is constructed and maintained in relation to one's communities [72]. As such, the self is logically separate and separable from the construct of the user. While the self refers to an individual's stable subjectivity across time [36], the 'user' variously refers to an individual's relationship with a device or the set of informational relationships emergent from the assemblage of 'user' and 'device' (e.g., [10]). From a humanistic perspective, the experience of the self supports the user that is constructed through interaction with technology: it is the mode of being an individual, a time-based, embodied mode of encountering the world through observable and consistent patterns of sensibility, intuition, and thought [37]. We are therefore faced with developing an approach to the user that is sufficiently constrained so as to be relevant, but not so reductionist as to be myopic in a technological sense (see e.g., [40]). We effect such a framing through the lens of social orientation.

Social orientation is theorized as a stable trait in people's modes of understanding themselves in relation to their worlds [72]. Traits are understood as intrinsic qualities of the self that remain invariant across contexts. States, by contrast, are transient manifestations of personality and are dependent upon context $[18,25]$. The contextdependency of specific privacy concerns could be considered analogous to states [67]. At the same time, digital privacy concerns could reflect a general tendency akin to a stable trait of the individual [67]. 
In individualist societies, people are autonomous and independent from the various communities within which they are situated. Individualists give priority to their personal goals over the goals of their communities and behave primarily on the basis of individual attitudes rather than community norms [71]. In contrast, in collectivist societies, "people are interdependent within their in-groups (family, tribe, nation, etc.), give priority to the goals of their in-groups, shape their behavior primarily on the basis of ingroup norms, and behave in a communal way" [71]. As Triandis and Gelfand [73] point out, the rough alignment of the individualism or collectivism of a given society with a national population is a gestalt effect. For example, people in the United States are not always individualists even though the United States is known to be an individualist society.

We contend that the imperative of community health sufficiently changes the context in which health-related mobile technologies are used. As a consequence, understanding the implications of population-level contact-tracing applications requires not just the lens of the individual, but also constructs related to the community. Given the theoretical relationship between the user and the self presented above, we contend that traits central to the self (i.e., social orientation) will predict the states demonstrated by the user (i.e., perceived benefit and expected use of pandemic-tracking apps), leading to the following hypotheses:

- H1a: The perceived benefit of pandemic-tracking apps is higher when the social framing used to describe such apps aligns with a person's own social orientation.

- H1b: The expected use of pandemic-tracking apps is higher when the social framing used to describe such apps aligns with a person's own social orientation.

Further, potential users are likely to perceive pandemic-tracking apps more favorably when those apps are framed in terms of outcomes that are most beneficial to them. In essence, potential users will think more favorably about an app and its use when that app does what it is supposed to do in the least intrusive way. Based on this, we formulated the following hypotheses:

- H2a: The perceived benefit of pandemic-tracking apps is higher with beneficial utility framing.

- H2b: The expected use of pandemic-tracking apps is higher with beneficial utility framing.

\subsection{Mobile Health}

There is a rich vein of mHealth research pertaining to mobile technologies and their social implications. For the most part, digital contact-tracing technologies have been developed to mitigate the impact of historically known infectious threats. These technologies are designed within a relatively stable sociotechnical context and are intended for use among a known and definable sub-population. Digital technologies have, for example, been deployed to combat influenza A (e.g., [28]), H1N1 (e.g., [62]), tuberculosis (e.g., [48]) and foot and mouth disease (e.g., [83]). In the case of rarer infectious agents (e.g., Ebola [1]), digital contact tracing was not proposed at a population scale as the result of a complex web of social, political, and economic circumstances. In contrast, population-level contract tracing can be a crucial tool for combating COVID-19, a pandemic that potentially affects every person in the world independent of socioeconomic status, citizenship, or lifestyle.

However, the benefits of mHealth research are most frequently framed in terms of individual users, rather than the communities to which those individuals belong [65]. In the context of the COVID-19 pandemic, the potential social implications of mHealth technologies have received both positive and negative assessments. Assessors have argued that digital contact tracing applications need not be creepy [13]. Critical calls have emerged from within surveillance studies, identifying a need to understand the ways in which digital contact tracing will contribute to surveillance culture [24]. Similar calls from within the HCI community highlight the need to systematically speculate about the relationship between Big Tech and pandemic-tracking technologies [22].

Wariness of mHealth in the context of the pandemic is, however, contextualized by a host of research that describes the benefits of mHealth. mHealth technologies present an opportunity to explore phronetic design, drawing on the everyday, practical experiences of users [11], thus combating the dehumanization of the user [17]. Researchers have demonstrated definite benefits of mHealth technologies across a range of patient populations, including but not limited to pregnant women [77], people with dietary concerns [49], and addicts [6]. Based on the apparent individual benefits of mHealth and the simultaneous disconnect between the individual and their communities identified in mHealth research [65], we see the potential relationship between users' social orientation and their privacy concerns about pandemic-tracking apps as a useful way of reframing mHealth at the intersection of society, community, and individual.

\subsection{Usable Privacy}

Privacy in the context of mobile technologies is the subject of a large body of work. Privacy concerns have been shown to be influenced by a diversity of aspects, such as mental models of app functionality (e.g., [26, 45]), motivations for sharing location-specific data (e.g., [53]), and expectations of the app or platform they use (e.g., [34]). Further, researchers have demonstrated that non-expert users, in general, do not have a nuanced understanding of how apps work and leverage folk theories to justify non-participation in expert-suggested security and privacy measures [76]. However, users show a tendency toward self-management of privacy when the option is available [7], and researchers have attempted to nudge users to engage in such self-management $[2,66]$.

Problematically, the efficacy of privacy self-management is not certain despite its centrality in much of the scholarly work on usable privacy (e.g. [5]). Indeed, privacy self-management is strained if not outright overburdened [64]. Its overburdening stems from end users' inability to manage heterogeneous and non-standardized privacy settings across the suite of apps they use [3]. Privacy concerns impact the use of novel technologies (e.g., [12, 27, 50, 69]) at the individual level. Individual use of pandemic-tracking apps is logically necessary for the achievement of community- and society-level pandemic mitigation. As such, we see the likely complex relationship between social orientation, privacy concerns, and perceptions of mHealth technologies as a productive in-road to understanding how individual perceptions can influence the achievement of 
collective good and, in turn, an emergent post-pandemic social contract.

\subsection{Mobile Health, Privacy Concerns, and the Pandemic}

It has been demonstrated that privacy concerns play a primary role in determining potential users' reported willingness to use mHealth devices $[54,55]$. The discourse of mHealth privacy in the United States is guided by the framing recommended by policy groups such as the National Committee for Vital and Health Statistics (NCVHS):

Health information privacy is an individual's right to control the acquisition, uses, or disclosures of his or her identifiable health data [21].

Ongoing work at the intersection of privacy and the pandemic broadly adheres to the approach to privacy described by NCVHS (e.g. $[33,44,56,59,80])$.

Redmiles [57] identified a preliminary list of six types of data as potentially relevant to privacy concerns regarding pandemictracking apps: (1) infection status, (2) level of exposure, (3) social graph, (4) locations, (5) (non-)use of the app, and (6) the infection statuses of the social group. Redmiles [57] further identified six parties capable of gaining access to data generated by pandemictracking apps. These include: (1) legitimate app users, (2) attackers who exploit the app, (3) the app maker (including employees of the app maker), (4) third party services used by the app (including those who work for organizations providing these services); (5) network providers, and (6) government entities that could obtain the data voluntarily or via legal means.

In other ongoing work, Cho et al. [19] consolidated these parties into three broad categories of external actants [42] relevant to privacy concerns and pandemic-tracking apps: snoopers (which Chan et al. [16] identify as malignant actors and Ma et al. [46] define as adversaries), contacts, and authorities. Bell et al. [14] reduced the category of "authorities" to the "government." However, it is just as likely that authorities might include the local police, emergency services, insurance companies, and healthcare systems, depending on the local context. "Contacts," is more of a term of art, however, being limited to its epidemiological operationalization [41, 68]: those with whom a user (i.e., a potential COVID-19 carrier) has come into contact.

Rather than focusing on specific forms of data that might be accessed by the contextually-changing 'Other' (i.e., government agencies, insurance companies, snoopers, etc.), our experiment relies less on the specificity of a priori taxonomies of data types and Others. We contend that users will engage in hyperbolic discounting [4] based on the immediate threat to health posed by COVID-19. Therefore, we anticipate the perceived immediate benefit of pandemic-tracking apps is likely to temper privacy concerns. However, we expect privacy concerns to correlate negatively with expected long-term use. We captured this aspect via the following hypotheses:

- H3a: The perceived benefit of pandemic-tracking apps is unrelated to privacy concerns.

- H3b: The expected use of pandemic-tracking apps is negatively related to privacy concerns.
Table 1: Number of responses in each treatment condition (Control Group: $n=43$ ).

\begin{tabular}{cccc}
\hline & & \multicolumn{2}{c}{ Social Framing } \\
& & Individualist & Collectivist \\
\hline Utility & Beneficial & 93 & 80 \\
Framing & Intrusive & 93 & 80 \\
\hline
\end{tabular}

\section{METHOD}

We conducted a questionnaire-based online experiment to examine the effect of social orientation, social framing, and utility framing on perceptions and expected use of pandemic-tracking technologies. In this section, we describe the study design, data collection, sample characteristics, and analysis plan. All study procedures were reviewed and approved by Indiana University Institutional Review Board (IRB).

\subsection{Study Design}

We developed a questionnaire that presented participants with the following scenario regarding the use of a fictive but realistic health-tracking technology in response to the outbreak of a novel contagious disease:

Core Scenario (Control): Based on a recommendation from the World Health Organization (WHO), you recently started using a smart thermometer app produced by one of several mainstream technology companies. The app gives you real-time information about the outbreak of a new and highly contagious virus, and using this app is proven to help you avoid becoming infected.

In order to see your likelihood of becoming infected and what recommended actions you can take to reduce this likelihood, you must provide the app with data every time you use it. The app records your temperature, prompts you to input your symptoms (if any), and uses a combination of Bluetooth technology and location data to measure your exposure to the virus.

In the treatment conditions, we appended additional text to the core scenario above. The additional text included information that we varied systematically based on social framing (individualist vs. collectivist) and utility framing (beneficial vs. intrusive), resulting in the $2 x 2$ experimental design shown in Table 1 . We characterize 'intrusiveness' as a scenario element that involves perceptions of boundary violations. However, the scenario text did not use the terms 'intrusion,' 'benefit,' 'individualist,' or 'collectivist' to avoid exposing participants to these terms because of their likely priming effects.

Specifically, the added text for each treatment condition was:

Individualist / Beneficial: A few days ago, you personally started using the WHO-recommended smart thermometer app. Ten days after starting to use the app, you have followed the app's recommended actions and remain uninfected. 
Individualist / Intrusive: A few days ago, you personally started using the WHO-recommended smart thermometer app. Ten days after starting to use the app, local emergency services inform you that the app data has flagged you for a 14-day quarantine and health monitoring.

Collectivist / Beneficial: A few days ago, everyone in your community started using the WHO-recommended smart thermometer app. Ten days after starting to use the app, the community has followed the app's recommended actions and the community infection rate is negligible.

Collectivist / Intrusive: A few days ago, everyone in your community started using the WHO-recommended smart thermometer app. Ten days after starting to use the app, local emergency services inform the community that the app data has flagged the community for a 14-day quarantine and health monitoring.

We varied social framing between subjects while utility framing was a within-subjects factor. In other words, participants in the treatment conditions encountered either two individualist or two collectivist scenarios, with one describing an outcome resulting in no quarantine and the other resulting in quarantine. The scenarios were presented in random order to counterbalance for potential order effects.

We constructed the scenarios through an iterative process of drafting and revision, drawing on contemporary news stories about pandemic-tracking applications. During the revision process, we incorporated feedback from colleagues regarding the realism and clarity of the scenarios. We adopted Meinert's [47] guidelines to make the scenarios realistic, multi-faceted, and open to interpretation (i.e., never 'right or wrong'). For example, we did not juxtapose the respective negative infection rates in the individualist and collectivist social framing conditions because it is not realistic that any app would be $100 \%$ successful at preventing infection for an entire community. Similarly, in the intrusive scenarios, the infection status is ambiguous thereby maintaining openness to interpretation as would likely be the case when using such an app in reality. Further, the scenarios contained passing references to Bluetooth and location data collection by the app in order to increase realism and openness to interpretation.

Figure 1 shows the overall organization of the study. Upon consent, we randomly assigned participants to either the individualist or collectivist social framing conditions. Within each, participants encountered two corresponding scenarios, one including the noquarantine outcome and other including the quarantine outcome. As mentioned earlier, the order of the two within-subjects scenarios was randomized to counterbalance for potential order effects.

After reading each scenario, participants answered scenariospecific questions, including two manipulation checks that tested attentive reading and comprehension of the scenario. Next, we provided participants with measures of privacy concerns and social orientation. We measured privacy concerns with the Mobile Users' Information Privacy Concern (MUIPC) scale [79] and social orientation via the Individualism-Collectivism (IND-COL) scale [73]. At the end, we collected basic demographics. The complete questionnaire is included in the Appendix (see Appendix Section A).

\subsection{Data Collection and Filtering}

We advertised the study as a Human Intelligence Task (HIT) on the AMT platform. AMT has been shown to serve as a suitable proxy for the segment of the United States population aged 18-50 with at least some college education [58] and to be useful in research addressing personal traits [20]. However, AMT samples do involve methodological risks, and research has shown that survey-based AMT studies should try to minimize the presence of the "faithless respondent" $[29,61]$. We included such precautions in the form of manipulation and attention checks embedded within the study. The attention checks directed participants to mark a specific answer to indicate attentive reading, and the manipulation checks required participants to answer two questions about the presented scenario.

We restricted participation to AMT workers based in the United States who had successfully completed at least 50 tasks with a task approval rating of $95 \%$ or higher. The study was available on AMT between April 22, 2020 and May 22, 2020, coinciding with the beginning of several mandatory, state-enforced 'shelter-in-place' orders within the United States in response to the COVID-19 pandemic as well as with the initial stages of a few states retracting those orders. Those who completed the study attentively were paid $\$ 1.50$. Based on a median study completion time of 12.7 minutes, the compensation translates to $\$ 7.09 /$ hour, which is in line with the state-specific minimum wage and is typical of AMT compensation rates.

Of the 955 responses received via AMT, we excluded 413 based on pre-determined criteria in line with best practices for mitigating the effects of inattentive participants [9]. Specifically, we excluded participants for the following reasons: (1) failure to pass either attention check included to ensure attentive participation (316 responses) and (2) failure to pass the manipulation check designed to confirm comprehension of the described scenario (97 responses). Note that we applied the exclusions serially in the order listed above. As a result, the 316 participants who failed one or both of the attention checks may additionally have failed one or both of the manipulation checks. It should be noted that manipulation checks serve as attention checks as well. Since we had confirmed readability of the scenarios in pre-study pilots that included non-native English speakers, we attribute the relatively high failure rate to 'faithless respondents' rather than the readability of the scenario. We excluded two additional responses because those participants skipped nearly all questions, including those related to our research questions. After filtering as described, we ended up with 540 valid responses.

\subsection{Sample and Data Analysis}

It should be noted that individualism and collectivism are not theoretically mutually exclusive, despite the apparent dichotomy created by the construct names. Just as a right-handed person can still perform tasks with the left hand, a primarily individualist person can demonstrate collectivist tendencies and vice versa. However, a lack of previous literature in this area precluded us from making specific hypotheses related to participants with mixed social orientations. Therefore, we focused exclusively on individualismdominant and collectivism-dominant participants. To that end, we separated 390 participants out of the 540 valid responses as being 


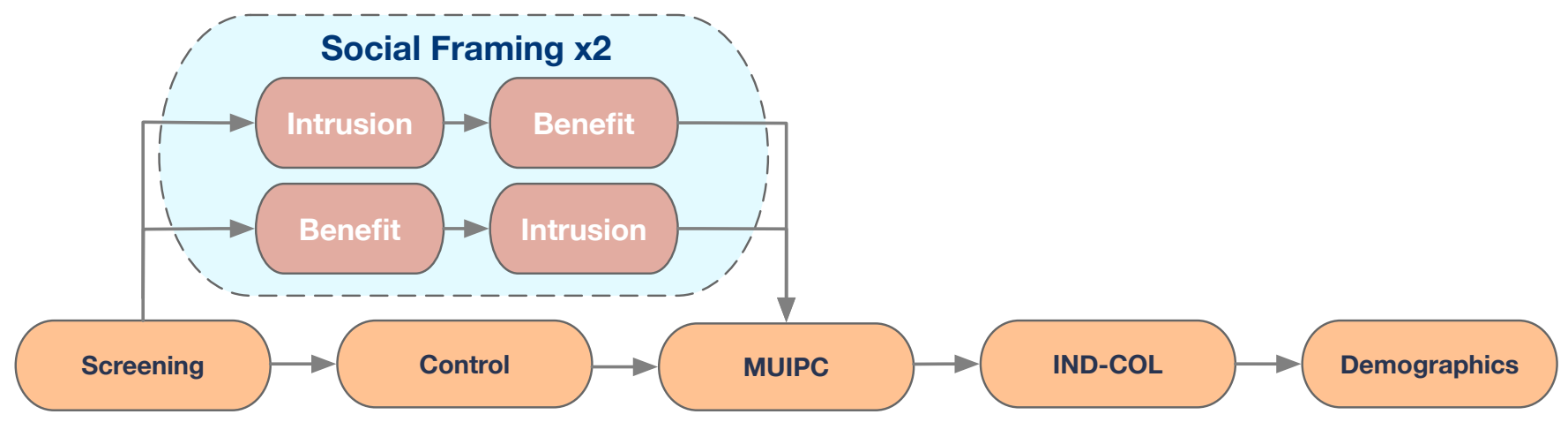

Figure 1: The overall flow of the study steps showing the various study conditions, with counterbalanced within-subjects utility framing presented in each between-subjects social framing treatment.

either individualism-dominant or collectivism-dominant. Participants were identified as individualism-dominant if their IND-COL responses indicated individualist social orientation and did not indicate a tendency toward collectivist traits (i.e., their scores on either of the collectivist sub-scales of the IND-COL did not meet the criteria to identify them as collectivists, but their scores on individualist sub-scales did identify them as individualists). Conversely, participants were identified as collectivism-dominant if their scores on the IND-COL did not indicate a tendency toward individualist traits. The analyses reported in the paper exclude the 150 participants who indicated possessing individualist as well as collectivist traits.

Within the individualism- and collectivism-dominant data set $(n=390), 260(66 \%)$ participants demonstrated the individualist social orientation, and $130(33 \%)$ participants demonstrated the collectivist social orientation. Of this group, $232(59 \%)$ specified their gender as male, $157(41 \%)$ as female, and one $(<1 \%)$ as non-binary. For statistical validity, the participant with non-binary gender was removed from analyses (since this individual would otherwise have been the sole member of a third level of the gender factor, making various analyses infeasible and/or invalid).

In the sub-sample used for the analyses reported in the paper $(n=389), 81$ participants $(21 \%)$ resided in rural areas, $181(47 \%)$ in the suburbs, and 127 (32\%) in cities. Participant ages ranged from 21 to 71, with a median of 36, mean of 39, and mode of 38 . Eleven participants (3\%) reported less than a high school education; eight $(2 \%)$ had earned high school diplomas; five (1\%) had received vocational training; 92 (24\%) indicated some college education; $194(50 \%)$ had completed a college degree, 66 (17\%) had a Master's degree; ten $(3 \%)$ had a doctoral degree; and three $(<1 \%)$ participants had professional degrees (e.g., MD, JD).

We tested H1a, H1b, H2a, and H2b using ordinal logistic regression (OLR) with cumulative link modeling (CLM). We calculated Spearman rank-order correlations to test $\mathrm{H} 3$ because the data is not normally distributed. CLM assessed the predictive value of several independent variables: social orientation (as measured by IND-COL), social framing, utility framing, age, and gender. Initial models included additional variables (i.e., parental status, political affiliation, prior use of digital health technologies, education). However, the additional variables were excluded from the final models because they failed scale effects tests as well as nominal effects tests, thus violating the assumptions of CLM. Our final models meet all assumptions of CLM.

\section{FINDINGS}

We present findings relative to the hypotheses stated in Section 2. We restate each hypothesis at the beginning of their respective subsection.

We describe several separate cumulative link models that test each of our hypotheses. These models demonstrate main effects and interaction effects of the predictor variables (i.e., social framing, utility framing, social orientation, age, and gender) on the four outcome variables (i.e., perceived benefit of pandemic-tracking apps, expected honest use of pandemic-tracking apps, expectation to take the infection-prevention measures recommended by pandemictracking apps, and expectation to continue using pandemic-tracking apps for general health monitoring after the pandemic). Following the presentation of results related to $\mathrm{H} 1 \mathrm{a}, \mathrm{H} 1 \mathrm{~b}, \mathrm{H} 2 \mathrm{a}$, and $\mathrm{H} 2 \mathrm{~b}$, we test H3a and H3b using Spearman correlation tests.

None of the variables considered in our models demonstrated collinearity according to the standard thresholds of $r=0.70$ [23] or $r=0.80$, which is common to epidemiological work [75]. Gender was not a statistically significant predictor in any of the models.

\subsection{Perceived Benefit}

H1a: The perceived benefit of pandemic-tracking apps is higher when the social framing used to describe such apps aligns with a person's own social orientation.

Table 2 presents the descriptive statistics for participants' response to the item, 'Using an app to monitor and control the spread of this outbreak is beneficial.' To determine whether social framing and social orientation predict participants' response to this item, we used the ordinal package in $\mathrm{R},{ }^{1}$ to fit a cumulative link model. The fit of this model is described by: log likelihood difference $=-14.41$; $\chi^{2}=28.81 ; d f=-8 ; p<0.001$.

To examine the statistical significance of predictors in our cumulative link model, we performed an analysis of deviance (ANODE), which is analogous to analysis of variance in linear models [78] (see Table 3 and Figure 2). We found that participants' social

\footnotetext{
${ }^{1}$ https://CRAN.R-project.org/package $=$ ordinal
} 
Table 2: Perceived benefit of pandemic-tracking apps, split across social orientation and social framing.

\begin{tabular}{ccccc}
\hline $\begin{array}{c}\text { Social } \\
\text { Framing }\end{array}$ & $\begin{array}{c}\text { Social } \\
\text { Orientation }\end{array}$ & n & Mean & SD \\
\hline Control & Collectivist & 20 & 5.20 & 1.77 \\
Individualist & Collectivist & 70 & 5.70 & 1.48 \\
Collectivist & Collectivist & 40 & 5.40 & 1.68 \\
Control & Individualist & 23 & 5.13 & 1.49 \\
Individualist & Individualist & 116 & 4.84 & 1.56 \\
Collectivist & Individualist & 120 & 5.09 & 1.59 \\
\hline
\end{tabular}

Table 3: Analysis of deviance for perceived benefit of pandemic-tracking apps.

\begin{tabular}{lrcc}
\hline Independent Variable & \multicolumn{1}{c}{$\chi^{2}$} & $\mathbf{d f}$ & $\mathbf{p}$ \\
\hline Social Framing (SF) & 0.36 & 2 & 0.83 \\
Social Orientation (SO) & 20.17 & 1 & $<0.0001$ \\
SF x SO & 4.15 & 2 & 0.12 \\
Age & 6.28 & 2 & 0.04 \\
Gender & 1.77 & 1 & 0.18 \\
\hline
\end{tabular}

orientation predicts perceived benefit of pandemic-tracking apps $\left(\chi^{2}(1)=20.17, p<0.0001\right)$. Collectivist participants perceived greater benefit to pandemic-tracking apps than did individualist participants. The average 7-point Likert-scale score for individualists $(n=259)$ was 4.981 (roughly 'Somewhat Agree') with a standard deviation of 1.566. The average response for collectivists $(n=130)$ was 5.531 (roughly 'Agree') with a standard deviation of 1.586. As Table 3 shows, we similarly found participant age to be a statistically significant predictor of the extent to which they perceive pandemictracking technologies to be beneficial $\left(\chi^{2}(2)=6.28, p=0.04\right)$. Older participants $(55+)$ perceived the lowest benefit from pandemictracking apps $(n=52$, mean $=4.67, s d=1.93)$, younger ones (under 35) indicated the highest benefit ( $n=160$, mean $=5.24, s d=$ 1.5 ), and the benefit perceived by those in the middle age group (35-54) fell in the middle $(n=177$, mean $=5.24, s d=1.59)$. The difference in perceived benefit between the middle age and oldest groups was statistically significant $(p=0.033)$, while the difference between the youngest and oldest groups was not $(p=0.11)$. The lack of statistical significance between the youngest and the oldest age groups could potentially be attributed to the difference in combined sample sizes and/or the conservative Tukey correction to avoid family-wise error. The main effect of social framing was not statistically significant $\left(\chi^{2}(2)=0.36, p=0.83\right)$, nor were interaction effects between social orientation and social framing $\left(\chi^{2}(2)=4.15, p=0.12\right)$.

In summary, we found that the perceived benefit of pandemictracking apps shows a main effect based on social orientation, with collectivists generally perceiving higher benefit. However, the perceived benefit is not influenced by the social framing used to describe pandemic-tracking apps nor by an interaction between social framing and social orientation. Therefore, we reject H1a.
Table 4: Expected honest use of pandemic-tracking apps, split across social orientation and social framing.

\begin{tabular}{ccccc}
\hline $\begin{array}{c}\text { Social } \\
\text { Framing }\end{array}$ & $\begin{array}{c}\text { Social } \\
\text { Orientation }\end{array}$ & n & Mean & SD \\
\hline Control & Collectivist & 20 & 5.95 & 0.95 \\
Individualist & Collectivist & 70 & 6.21 & 1.13 \\
Collectivist & Collectivist & 40 & 5.78 & 1.39 \\
Control & Individualist & 23 & 5.65 & 1.19 \\
Individualist & Individualist & 116 & 5.05 & 1.71 \\
Collectivist & Individualist & 120 & 5.21 & 1.54 \\
\hline
\end{tabular}

Table 5: Analysis of deviance for expected honest use of pandemic-tracking apps.

\begin{tabular}{lrcc}
\hline Independent Variable & \multicolumn{1}{c}{$\chi^{2}$} & $\mathbf{d f}$ & $\mathbf{p}$ \\
\hline Social Framing (SF) & 0.87 & 2 & 0.65 \\
Social Orientation (SO) & 29.30 & 1 & $<0.0001$ \\
SF x SO & 6.84 & 2 & 0.03 \\
Age & 2.30 & 2 & 0.32 \\
Gender & 1.09 & 1 & 0.30 \\
\hline
\end{tabular}

\subsection{Expected Use}

H1b: The expected use of pandemic-tracking apps is higher when the social framing used to describe such apps aligns with a person's own social orientation.

We examined the expected use of pandemic-tracking apps in terms of three facets of expected use by repeating the CLM analyses reported above for H1a. The following subsections describe the analyses for each expected-use facet in turn.

4.2.1 Expected Honest Use. Table 4 presents the descriptive statistics for responses to the item, 'When using this app, I would provide honest and accurate information about my symptoms.' The CLM that demonstrates the predictive value of social orientation, social framing, age, and gender is described by: log likelihood difference $=-22.25 ; \chi^{2}=44.50 ; d f=-8 ; p<0.0001$.

We performed an ANODE to examine the statistical significance of predictors in the CLM (see Table 5). We found that social orientation accounts for a statistically significant deviance in expected honest use of pandemic-tracking apps $\left(\chi^{2}(1)=29.30, p<0.0001\right)$. On a seven-point scale, the average score for individualists $(n=259)$ was 5.18 (roughly 'Somewhat Agree') with a standard deviation of 1.60 , while that for collectivists $(n=130)$ was 6.04 (roughly 'Agree') with a standard deviation of 1.20 . Social framing did not exhibit a main effect $\left(\chi^{2}(2)=0.87, p=0.65\right)$, nor did age or gender. However, the interaction effect between social framing and social orientation was statistically significant $\left(\chi^{2}(2)=6.84, p=0.03\right)$.

We further examined the interaction effect with a post-hoc test for differences in least square means using the Tukey method to control for family-wise error. The difference between collectivist participants in the individualist social framing condition (mean $=6.21$ [roughly 'Agree'], $s d=1.13$ ) and individualist participants in the 


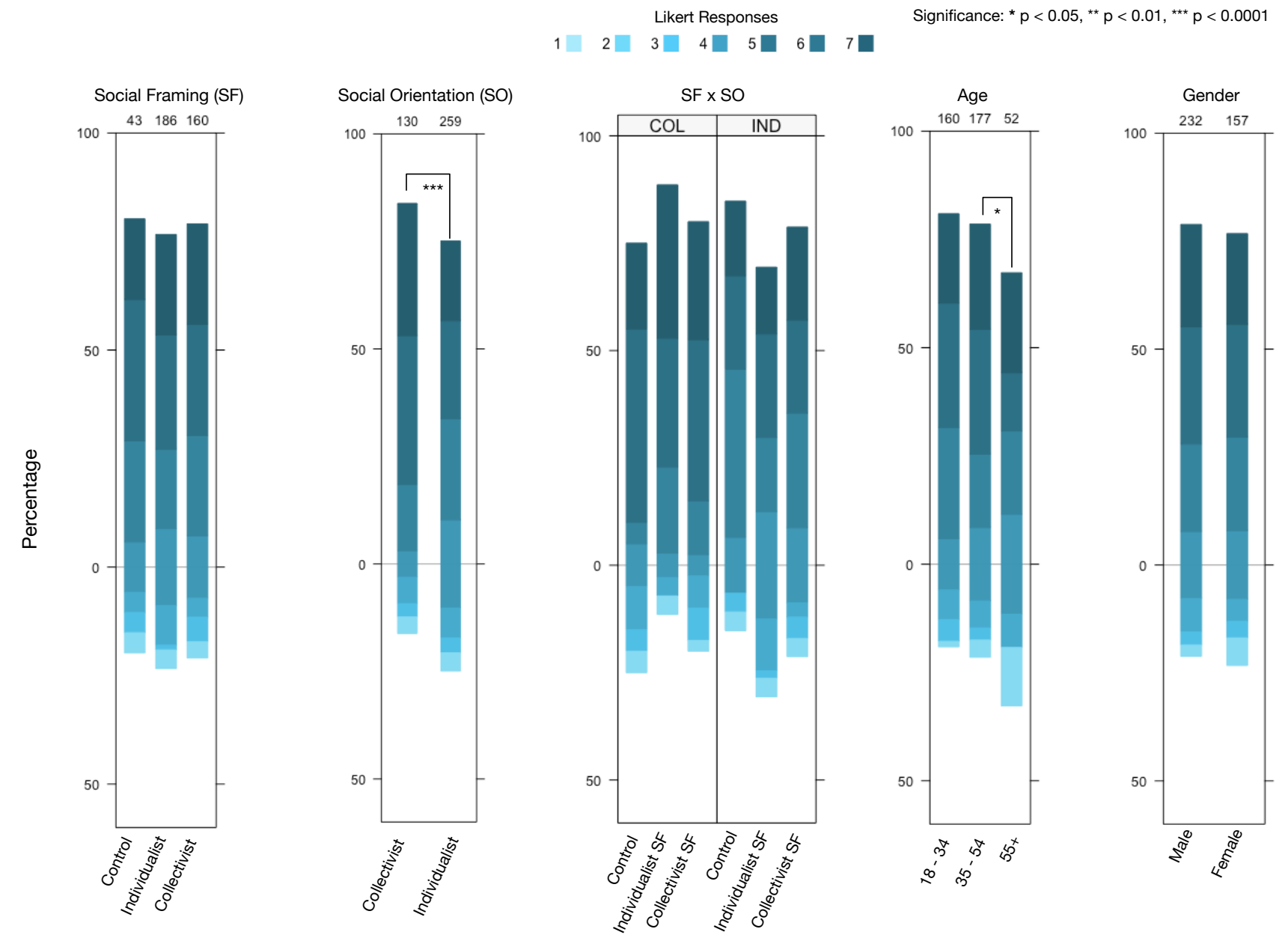

Figure 2: Results of the CLM for perceived benefit of pandemic-tracking apps. The results show that social orientation and age are statistically significant predictors of perceived benefit.

individualist social framing condition (mean $=5.05$ [roughly 'Somewhat Agree'], $s d=1.71)$ was statistically significant $(p<0.0001)$. The difference between the same collectivist participants and individualists in the collectivist social framing condition (mean $=5.21$ [roughly 'Somewhat Agree'], $s d=1.54$ ) was statistically significant as well $(p<0.0001)$. Figure 3 shows a graphical representation of these results.

The above interaction effect between social orientation and social framing indicates that collectivists in the individual social framing condition differ from individualists in both social framing conditions regarding expected honest use of pandemic-tracking apps. Since H1b posits a comparison within the collectivist and individualist participant groups based on congruence between the social orientation and social framing, we reject $\mathrm{H} 1 \mathrm{~b}$ as it pertains to expected honest use of pandemic-tracking apps.

4.2.2 Expectation to Take App-Recommended Actions. Table 6 presents the descriptive statistics for responses to the item, 'I would take the infection-prevention measures recommended by the app.' The CLM that demonstrates the predictive value of social orientation, social framing, age, and gender is described by: log likelihood difference $=-24.88 ; \chi^{2}=49.76 ; d f=-8 ; p<0.0001$.

Table 7 presents the results of ANODE testing, revealing that social orientation accounts for a statistically significant deviance in the expectation to take the infection-prevention measures recommended by pandemic-tracking apps $\left(\chi^{2}(1)=38.53, p<0.0001\right)$. On a 7-point scale, the average score for individualists $(n=260)$ was 5.10 (roughly 'Somewhat Agree') with a standard deviation of 1.44 while that for collectivists $(n=130)$ was 5.94 (roughly 'Agree') with a standard deviation of 1.21. Social framing did not exhibit a main effect $\left(\chi^{2}(2)=2.69, p=0.26\right)$, nor did age or gender. However, the interaction effect between social framing and social orientation was statistically significant $\left(\chi^{2}(2)=6.90, p=0.03\right)$.

We further examined the interaction effect with a post-hoc test for differences in least square means using the Tukey method to control for family-wise error. As represented in Figure 4, we 


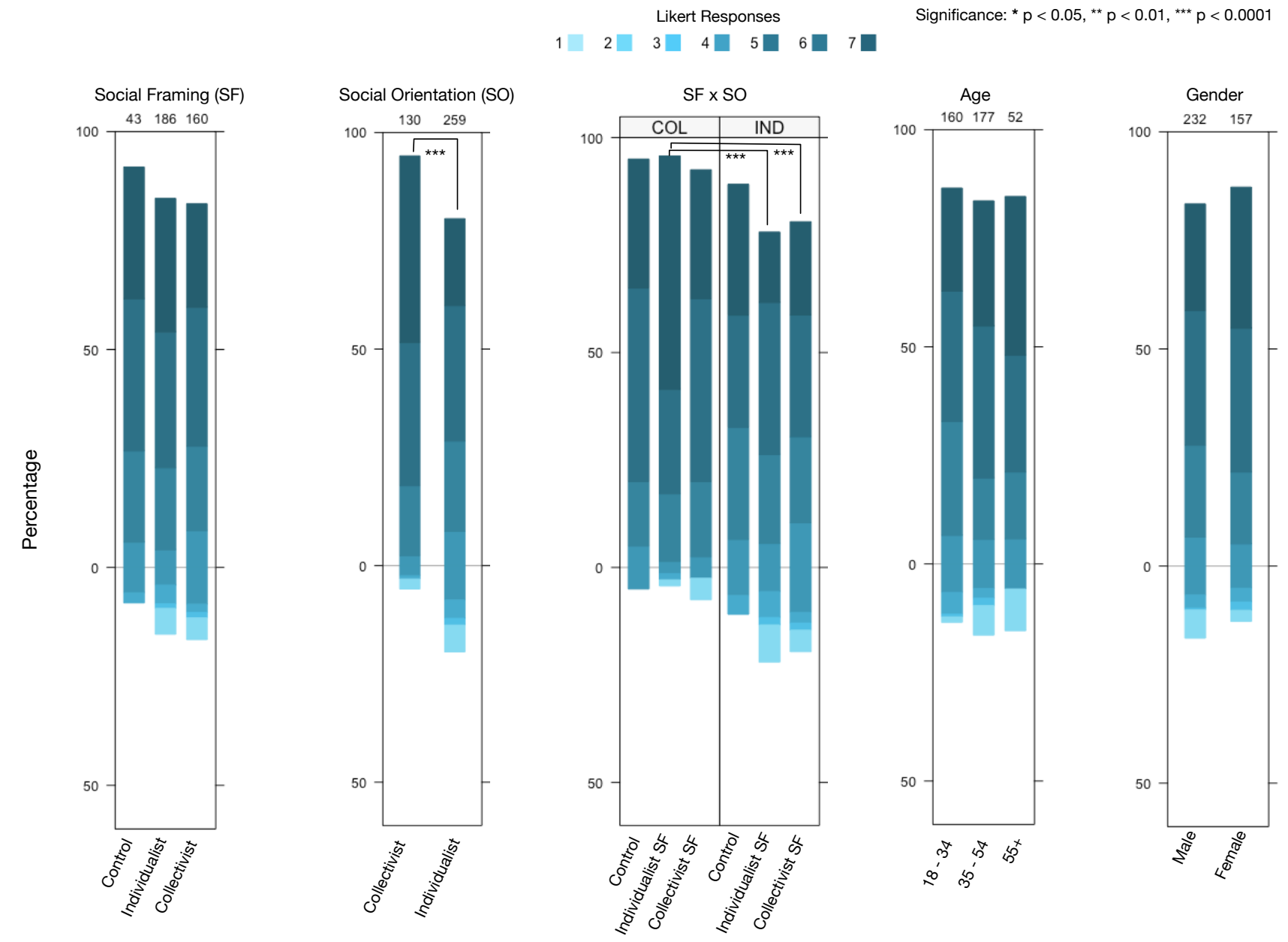

Figure 3: Results of the CLM for expected honest use of pandemic-tracking apps. The results show that social orientation and interaction between social orientation and social framing are statistically significant predictors of expected honest use.

Table 6: Expectation to take the infection-prevention measures recommended by pandemic-tracking apps, split across social orientation and social framing.

\begin{tabular}{ccccc}
\hline $\begin{array}{c}\text { Social } \\
\text { Framing }\end{array}$ & $\begin{array}{c}\text { Social } \\
\text { Orientation }\end{array}$ & n & Mean & SD \\
\hline Control & Collectivist & 20 & 5.30 & 1.66 \\
Individualist & Collectivist & 70 & 6.13 & 1.13 \\
Collectivist & Collectivist & 40 & 5.93 & 0.10 \\
Control & Individualist & 23 & 5.00 & 1.35 \\
Individualist & Individualist & 116 & 4.94 & 1.43 \\
Collectivist & Individualist & 120 & 5.27 & 1.45 \\
\hline
\end{tabular}

found statistically significant differences between the following pairs of participant groups: (i) collectivists in the individualist social framing condition $(n=70$, mean $=6.13, s d=1.13)$ and
Table 7: Analysis of deviance for expectation to take the infection-prevention measures recommended by pandemictracking apps.

\begin{tabular}{lrcc}
\hline Independent Variable & \multicolumn{1}{c}{$\chi^{2}$} & $\mathbf{d f}$ & $\mathbf{p}$ \\
\hline Social Framing (SF) & 2.69 & 2 & 0.26 \\
Social Orientation (SO) & 38.53 & 1 & $<0.0001$ \\
SF x SO & 6.90 & 2 & 0.03 \\
Age & 4.34 & 2 & .0 .11 \\
Gender & 0.10 & 1 & 0.92 \\
\hline
\end{tabular}

individualists in the control condition $(n=23$, mean $=5, s d=1.35)$ $(p<0.001)$; (ii) collectivists in the individualist social framing condition $(n=70$, mean $=6.13, s d=1.13)$ and individualists in the individualist social framing condition $(n=116$, mean $=4.94, s d=1.43)$ 
Table 8: Expected continued use of pandemic-tracking apps, split across social orientation and social framing.

\begin{tabular}{ccccc}
\hline $\begin{array}{c}\text { Social } \\
\text { Framing }\end{array}$ & $\begin{array}{c}\text { Social } \\
\text { Orientation }\end{array}$ & n & Mean & SD \\
\hline Control & Collectivist & 20 & 4.00 & 1.95 \\
Individualist & Collectivist & 70 & 4.97 & 1.73 \\
Collectivist & Collectivist & 40 & 4.75 & 1.98 \\
Control & Individualist & 23 & 3.74 & 1.57 \\
Individualist & Individualist & 116 & 4.26 & 1.71 \\
Collectivist & Individualist & 120 & 4.30 & 1.85 \\
\hline
\end{tabular}

( $p<0.0001)$; (iii) collectivists in the individualist social framing condition $(n=70$, mean $=6.13, s d=1.13)$ and individualists in the collectivist social framing condition $(n=120$, mean $=5.27, s d=1.45)$ ( $p=0.0001$ ); and (iv) collectivists in the collectivist social framing condition $(n=40$, mean $=5.93, s d=0.10)$ and individualists in the individualist social framing condition $(n=116$, mean $=4.94, s d=$ 1.43) $(p=0.0015)$. We found no other statistically significant differences.

The above interaction effect between social orientation and social framing indicates that collectivists in the individual social framing condition differ from individualists in both social framing conditions as well as the control condition regarding taking actions recommended by pandemic-tracking apps. Since, H1b posits a comparison within the collectivist and individualist participant groups based on congruence between the social orientation and social framing, we reject $\mathrm{H} 1 \mathrm{~b}$ as it pertains to expectations to take actions recommended by pandemic-tracking apps. However, when there was congruence between social framing and social orientation, collectivists were more likely to indicate taking the app-recommended actions than individualists.

4.2.3 Expected Continued Use. Table 8 presents the descriptive statistics for responses to the item, 'If possible, I would continue to monitor my health using this app after the outbreak has been controlled.' The CLM that demonstrates the predictive value of social orientation, social framing, age, and gender is described by: $\log$ likelihood difference $=-13.44 ; \chi^{2}=26.88 ; d f=-8 ; p<$ 0.0001 .

To examine the statistical significance of predictors in our CLM, we performed an ANODE. Table 9 presents the results of this ANODE. We found three statistically significant main effects, but no interaction effects. We describe them below and present them graphically in Figure 5. We did not find a statistically significant interaction effect between social orientation and social framing $\left(\chi^{2}(2)=0.35, p=0.84\right)$.

We performed an ANODE to examine the statistical significance of predictors in the CLM, finding three statistically significant effects (see Table 9 and Figure 5). First, there was a main effect for social orientation $\left(\chi^{2}(1)=13.54, p<0.001\right)$. On a seven-point scale, the average for individualists $(n=260)$ was 4.22 (roughly 'Neither Agree nor Disagree') with a standard deviation of 1.77, while that for collectivists ( $n=130$ ) was 4.75 (roughly 'Somewhat Agree') with a standard deviation of 1.86. Second, we found a main effect for social framing $\left(\chi^{2}(2)=7.78, p=0.02\right)$. On a seven-point scale, the
Table 9: Analysis of deviance for expected continued use of pandemic-tracking apps.

\begin{tabular}{lrcc}
\hline Independent Variable & \multicolumn{1}{c}{$\chi^{2}$} & $\mathbf{d f}$ & $\mathbf{p}$ \\
\hline Social Framing (SF) & 7.78 & 2 & 0.02 \\
Social Orientation (SO) & 13.54 & 1 & $<0.001$ \\
SF x SO & 0.36 & 2 & 0.84 \\
Age & 7.97 & 2 & 0.02 \\
Gender & 1.13 & 1 & 0.29 \\
\hline
\end{tabular}

average response for participants in the individualist social framing condition $(n=186)$ was 4.53 (roughly, 'Somewhat Agree') with a standard deviation of 1.74, that for those in the collectivist social framing condition $(n=160)$ was 4.41 (roughly, 'Neither Agree nor Disagree') with a standard deviation of 1.90 , and that for those in the control group $(n=43)$ was 3.86 (roughly 'Neither Agree nor Disagree') with a standard deviation of 1.74 . Third, we found a main effect for age $\left(\chi^{2}(2)=7.97, p=0.02\right)$ with those in the 18-34 age group $(n=160$, mean $=4.67, s d=1.73)$ being slightly more likely to continue using the app after the pandemic than those aged 35-55 $(n=177$, mean $=4.23, s d=1.88)$.

Based on this model, we conclude that social framing, social orientation, and age each predict expected continued use of pandemictracking apps after the pandemic. However, the absence of a significant interaction effect leads us to reject $\mathrm{H} 1 \mathrm{~b}$ as it pertains to expected continued use of pandemic-tracking apps after the pandemic.

\subsection{Utility Framing and Privacy Concerns}

H2a: The perceived benefit of pandemic-tracking apps is higher with beneficial utility framing.

H2b: The expected use of pandemic-tracking apps is higher with beneficial utility framing.

To examine the extent to which utility framing predicts participant agreement with statements about the perceived benefit and expected use of pandemic-tracking apps, we fitted separate CLMs using responses as the outcome variable and utility framing, gender, and age as predictor variables, respectively. Since utility framing was not a statistically significant predictor in any of the models, we reject hypotheses $\mathrm{H} 2 \mathrm{a}$ and $\mathrm{H} 2 \mathrm{~b}$.

H3a: The perceived benefit of pandemic-tracking apps is unrelated to privacy concerns.

H3b: The expected use of pandemic-tracking apps is negatively related to privacy concerns.

To test the relationship between privacy concerns and perceived benefit of pandemic-tracking apps (H3a), we calculated corresponding Spearman correlations with Holm-Bonferroni corrections to mitigate family-wise error. We used Spearman correlation because the responses to these variable are not normally distributed. The correlations pertain to the relationship between participant agreement with the statement, 'Using an app to monitor and control the spread of this outbreak is beneficial' and the MUIPC constructs of perceived surveillance $(r=-0.06, p=0.22)$, perceived intrusion $(r=-0.07, p=0.19)$, and secondary use of personal information 


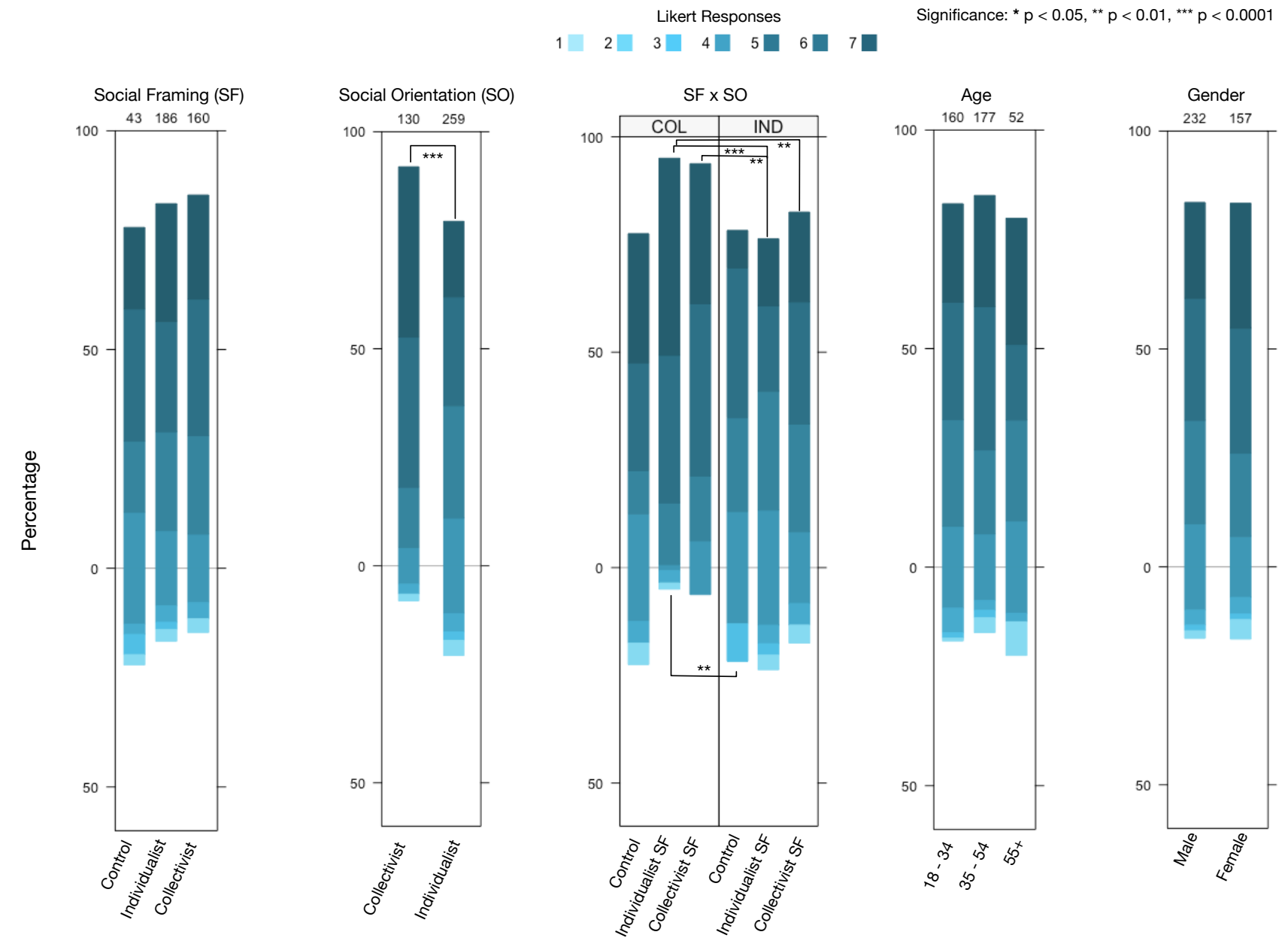

Figure 4: Results of the CLM for expectation to take the infection-prevention measures recommended by pandemic-tracking apps. The results show that social orientation and interaction between social framing and social orientation are statistically significant predictors of expectation to take app-recommended actions.

$(r=-0.08, p=0.10)$, respectively. The lack of statistically significant correlations between perceived benefit and privacy concerns as measured by the three constructs within the MUIPC scales seems to confirm H3a.

For H3b, we similarly found no statistically significant Spearman correlations between the MUIPC constructs of perceived surveillance, perceived intrusion, and secondary use of personal information and two of the facets of expected use: expected honest use $(r=0.04, p=0.42 ; r=0.02, p=0.71 ; r=0.04, p=0.47$, respectively) and expectation to take actions recommended by the app $(r=0.07, p=0.16 ; r=0.03, p=0.58 ; r=0.06, p=0.26$, respectively). However, we found that expected continued use of the app after the pandemic was statistically significantly negatively correlated with all three constructs related to privacy concerns: perceived surveillance $(r=-0.23, p<0.0001)$, perceived intrusion $(r=-0.21, p<0.0001)$, and secondary use of personal data $(r=-0.25, p<0.0001)$. These results indicate that expected postpandemic use of pandemic-tracking apps is indeed negatively influenced by privacy concerns as hypothesized in H3b. However, the negative influence of privacy concerns is unrelated to whether the expected use will involve honest reporting of symptoms and taking app-recommended actions.

In essence, our findings indicate that short-term considerations during the pandemic may lead people to relax privacy concerns temporarily. However, the temporary relaxation of privacy concerns does not extend to the use of the app after the pandemic is over.

\section{DISCUSSION}

We discuss the relevance of our findings to the present state of the pandemic and proceed to point out their significance for the post-pandemic social contract. 


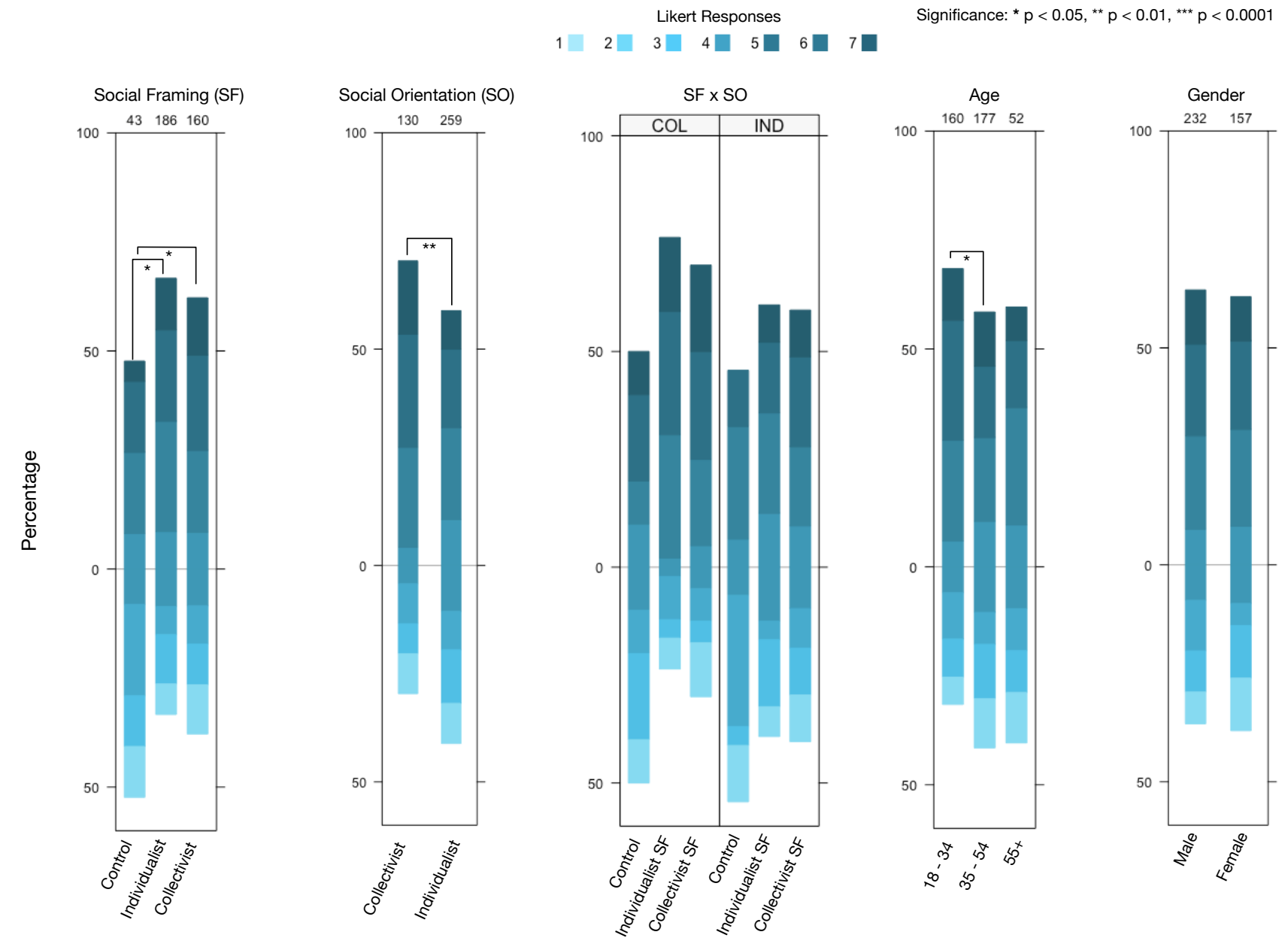

Figure 5: Results of the CLM for expected continued use of pandemic-tracking apps. The results show that social framing, social orientation, and age are statistically significant predictors of expected continued use.

\subsection{The Present: Social Orientation, Perceptions, and Privacy Concerns}

Perceived benefit and expected use of pandemic-tracking apps are impacted by social orientation, social framing, and privacy concerns in various ways. Therefore, taking such social factors into account is particularly important when designing and deploying pandemictracking applications.

We interpret the role of collectivist social orientation in perceptions and expectations of pandemic-tracking apps in terms of group preservation. Compared to individualists, collectivist participants indicated higher perceived benefit and expected use of pandemic-tracking apps. We interpret the difference as an effect of the relationship between the collectivist social orientation and a person's derivation of 'self' because collectivists derive their sense of self from membership in groups [71]. The threat to health posed by the pandemic risks destroying the composition of groups and communities, thus endangering the collectivist sense of self.
In the case of collectivist participants in the individualist social framing treatment, the bodily threats of the pandemic appear to act as an intensifier, with the heightened concern for one's own bodily health adding to the already positive perception of the benefit of pandemic-tracking apps. Such an interaction between collectivist social orientation and individualist social framing possibly indicates that individualist framing further boosts collectivist reliance on groups for the formation of identity as well as the biological facet of self-preservation upon which the maintenance of identity is ultimately predicated.

Unfortunately, the converse was not true: collectivist social framing had no effect on perceived benefit or expected use of pandemictracking apps indicated by individualists. The lack of a difference indicates that improving individualist perceptions and expected use of pandemic-tracking apps will be difficult. However, such improvement may be necessary given the extent to which the virus has remained uncontrolled in the United States, a notably individualist society [63]. The individualist nature of the United States society 
necessitates the achievement of collective good from individualist motivations. Therefore, achieving collective public health outcomes using pandemic-tracking technologies might be difficult because individualists demonstrate lower perceived benefit and expected use of pandemic-tracking apps and are unaffected by collectivist social framing. In light of our findings, we further contend that it is necessary to develop communication and outreach strategies that take into account the likely influence of underlying social orientations of individuals and societies.

The lack of a statistically significant relationship between privacy concerns and perceived benefit of pandemic-tracking apps is in line with prior research indicating that health issues override privacy [82]. We posit that the threat to health posed by the pandemic renders technological solutions immune to immediate privacy concerns. While pandemic-tracking apps do evoke privacy concerns, these take a back seat to the more pressing issues of bodily health. However, the secondary attention to privacy concerns is limited to the present, reappearing within a general long-term perspective.

\subsection{The Future: Privacy Concerns and Continued Use}

The technologies we develop today may become the infrastructures of tomorrow. Expected continued use of pandemic-tracking apps serves as a means of understanding their potential routinization. Given the overall slight tendency to expect to continue using pandemic-tracking apps after the pandemic, we see likely routinization of apps as a form of general health monitoring. Individualists, in particular, were ambivalent about continuing to use pandemictracking apps, indicating potential trepidation regarding long-term ramifications of routine use.

We contend that long-term privacy concerns form the roots of such worries. As we discovered, expected continued use showed statistically significant negative correlations with all three facets of privacy concerns (i.e., perceived surveillance, perceived intrusion, and secondary use of personal data). Despite these worries, younger participants indicated statistically significantly higher willingness to continue using pandemic-tracking apps for general health monitoring after the pandemic. The interplay of these contrasting findings points to an emergent social contract that is fraught with tension. On the one hand, we found a general negative relationship between privacy concerns and expected continued use of pandemictracking apps. On the other hand, younger users are inclined to continue using such apps for general health monitoring. While there is some inclination to continue using pandemic-tracking apps for general health-monitoring, there is also concern regarding how that use will impact privacy.

The immediate and pressing need to control the pandemic holds long-term implications for the social contract. Our findings highlight that such implications will be founded partially on the differences between near-term and long-term privacy concerns. Given that the temporary relaxation of privacy concerns in the immediate term does not seem to extend to everyday use in the long term, routinizing practices based only on the lowered immediate privacy concerns risks a post-pandemic social contract fraught with tension.

\section{IMPLICATIONS}

Our findings demonstrate that social orientation is a meaningful factor related to the perceptions and expected use of pandemictracking technologies. Therefore, it might be useful to include social orientation when investigating the perception and adoption of other potentially infrastructural technologies. Moreover, social orientation may be of relevance to the field of usable privacy in general.

Our research highlights the relatively stable perception of benefit and expected use of pandemic-tracking apps within the individualist and collectivist subgroups. As a result of the stability, promoting collective good by employing a collectivist message framing has limited effect on collectivists because it is preaching to the converted.

Individualists, in particular, seem to maintain views that are mostly unaffected by framing. Therefore, if pandemic-tracking apps are relied upon as a means of pandemic mitigation in the individualist-dominant United States, it would be necessary to frame them via messaging that leverages self-interest. However, we found that the individualist social framing we used did not have the intended effect, suggesting that we need to develop modes of communication that speak directly to the core characteristics of individualist social orientation in stronger and more explicit ways.

\section{LIMITATIONS AND FUTURE WORK}

Our study is limited to the United States and constrained by the limitations of self-selection, self-reporting, and the composition of the AMT worker population [58]. Since privacy concerns and public health matters can vary across countries, cultures, and subpopulations, additional studies are needed to verify the extent to which our findings generalize to other populations as well as to other operationalizations of 'community.'

The findings are based on attitudes expressed at a specific point during the ongoing COVID-19 pandemic. A longitudinal investigation is needed to understand the impact of temporally changing factors, such as infection rates, lockdowns, media coverage, government response, deployment and adoption of pandemic-related technologies, etc. Longitudinal investigations can further track shifts in attitudes as the immediate strife of the pandemic subsides.

\section{CONCLUSION}

We found that social orientation is a consistent statistically significant predictor of perceived benefit and expected use of pandemictracking apps, with collectivists indicating greater benefit and expected use compared to individualists. Moreover, individualists' perceptions of such apps appear to be independent of whether the app is framed in individualist or collectivist terms. In an individualistdominant society such as the United States, this can be problematic for increasing the adoption of pandemic-tracking apps since we cannot rely on the "better angels of [individualists'] nature" to increase adoption of pandemic-tracking technologies.

While it may be possible to improve the perceptions of pandemictracking apps through carefully crafted messaging and design, such improvements should not be undertaken with a blind eye to future privacy concerns. Perhaps unsurprisingly, we found that privacy concerns are relegated to secondary status in light of the immediate 
health threats of COVID-19. Yet, privacy concerns do emerge as a significant factor when the use of health-tracking apps is envisioned as infrastructure for general health monitoring after the pandemic has ended. As we move into a post-pandemic future, we are ethically obligated to understand not only the pressing need to control the pandemic now, but the impact that techno-solutionist approaches may have on the post-pandemic social contract in the longer term. Therefore, it is essential that the broader sociocultural and humancentered impacts of techno-solutionist approaches to controlling COVID-19 be considered not as an afterthought or a matter to be dealt with later, but as a foundation for the solutions we innovate. Collective good is possible only when we design, build, and use for the collective. It is through such consideration that we might achieve a social contract that is framed not by 'us, them, and it' but by 'we,' grounded in the condition of experiential subjectivity that binds us all.

\section{ACKNOWLEDGMENTS}

We thank the participants of our study. We are grateful to Kostas Gemenis, Ylva Ferstl, and Bart P. Knijnenburg for consultation regarding statistical analyses and graphics. Morgan L. Brockman helped with managing data collection via Amazon Mechanical Turk. This research was supported by a grant from Indiana University Office of the Vice President for Research. The contents of the paper are the work of the authors and do not necessarily reflect the views of the sponsors.

\section{REFERENCES}

[1] Solomon O. Abiola, Eric Portman, Henry Kautz, and E. Ray Dorsey. 2015. Node view: A mHealth real-time infectious disease interface - 2014 Ebola outbreak case study. In Adjunct Proceedings of the 2015 ACM International Foint Conference on Pervasive and Ubiquitous Computing and Proceedings of the 2015 ACM International Symposium on Wearable Computers (Osaka, Japan) (UbiComp/ISWC '15 Adjunct). Association for Computing Machinery, New York, NY, USA, 297-300. https: //doi.org/10.1145/2800835.2800851

[2] Alessandro Acquisti, Idris Adjerid, Rebecca Balebako, Laura Brandimarte, Lorrie Faith Cranor, Saranga Komanduri, Pedro Giovanni Leon, Norman Sadeh, Florian Schaub, Manya Sleeper, et al. 2017. Nudges for privacy and security: Understanding and assisting users' choices online. ACM Computing Surveys (CSUR) 50, 3 (2017), 1-41.

[3] Alessandro Acquisti, Laura Brandimarte, and George Loewenstein. 2015. Privacy and human behavior in the age of information. Science 347, 6221 (2015), 509-514.

[4] Alessandro Acquisti and Jens Grossklags. 2003. Losses, gains, and hyperbolic discounting: An experimental approach to information security attitudes and behavior. In 2nd Annual Workshop on Economics and Information Security-WEIS, Vol. 3. WEIS, Berkeley, CA, 1-27.

[5] Alessandro Acquisti and Jens Grossklags. 2005. Privacy and rationality in individual decision making. IEEE Security \& Privacy 3, 1 (2005), 26-33.

[6] Roy Aizen, Gabriela Marcu, Anjali Misra, Gregory Sieber, David G. Schwartz Alexis Roth, and Stephen Lankenau. 2018. Designing an emergency response community for opioid overdoses in Philadelphia. In Extended Abstracts of the 2018 CHI Conference on Human Factors in Computing Systems (Montreal QC, Canada) (CHI EA '18). Association for Computing Machinery, New York, NY, USA, 1-6. https://doi.org/10.1145/3170427.3188581

[7] Hazim Almuhimedi, Florian Schaub, Norman Sadeh, Idris Adjerid, Alessandro Acquisti, Joshua Gluck, Lorrie Faith Cranor, and Yuvraj Agarwal. 2015. Your location has been shared 5,398 times! A field study on mobile app privacy nudging. In Proceedings of the 33rd Annual ACM Conference on Human Factors in Computing Systems (Seoul, Republic of Korea) (CHI '15). Association for Computing Machinery, New York, NY, USA, 787-796. https://doi.org/10.1145/2702123.2702210

[8] Samuel Altmann, Luke Milsom, Hannah Zillessen, Raffaele Blasone, Frederic Gerdon, Ruben Bach, Frauke Kreuter, Daniele Nosenzo, Severine Toussaert, and Johannes Abeler. 2020. Acceptability of app-based contact tracing for COVID-19: Cross-country survey evidence. https://doi.org/10.1101/2020.05.05.20091587 Preprint, medRxiv.

[9] R. Michael Alvarez, Lonna Rae Atkeson, Ines Levin, and Yimeng Li. 2019. Paying attention to inattentive survey respondents. Political Analysis 27, 2 (2019), 145162. https://doi.org/10.1017/pan.2018.57
[10] Jeffrey Bardzell and Shaowen Bardzell. 2015. The user reconfigured: On subjectivities of information. In Proceedings of The Fifth Decennial Aarhus Conference on Critical Alternatives (Aarhus, Denmark) (CA '15). Aarhus University Press, Aarhus N, 133-144. https://doi.org/10.7146/aahcc.v1i1.21298

[11] Marguerite Barry, Kevin Doherty, Jose Marcano Belisario, Josip Car, Cecily Morrison, and Gavin Doherty. 2017. mHealth for maternal mental health: Everyday wisdom in ethical design. In Proceedings of the 2017 CHI Conference on Human Factors in Computing Systems (Denver, Colorado, USA) (CHI '17). Association for Computing Machinery, New York, NY, USA, 2708-2756. https://doi.org/10.1145/3025453.3025918

[12] Eric P.S. Baumer, Phil Adams, Vera D. Khovanskaya, Tony C. Liao, Madeline E. Smith, Victoria Schwanda Sosik, and Kaiton Williams. 2013. Limiting, leaving, and (re)lapsing: An exploration of Facebook non-use practices and experiences. In Proceedings of the SIGCHI Conference on Human Factors in Computing Systems (Paris, France) (CHI '13). Association for Computing Machinery, New York, NY, USA, 3257-3266. https://doi.org/10.1145/2470654.2466446

[13] Genevieve Bell. 2020. We need mass surveillance to fight COVID-19 - but it doesn't have to be creepy. https://www.technologyreview.com/2020/04/12/ 999186/covid-19-contact-tracing-surveillance-data-privacy-anonymity/ MIT Technology Review, May 2020.

[14] James Bell, David Butler, Chris Hicks, and Jon Crowcroft. 2020. Tracesecure: Towards privacy preserving contact tracing. arXiv:2004.04059.

[15] Rafael A Calvo, Sebastian Deterding, and Richard M Ryan. 2020. Health surveillance during COVID-19 pandemic. https://doi.org/10.1136/bmj.m1373

[16] Justin Chan, Shyam Gollakota, Eric Horvitz, Joseph Jaeger, Sham Kakade, Tadayoshi Kohno, John Langford, Jonathan Larson, Sudheesh Singanamalla, Jacob Sunshine, et al. 2020. PACT: Privacy sensitive protocols and mechanisms for mobile contact tracing. arXiv:2004.03544

[17] Stevie Chancellor, Eric P. S. Baumer, and Munmun De Choudhury. 2019. Who is the "human" in human-centered machine learning: The case of predicting mental health from social media. Proc. ACM Hum.-Comput. Interact. 3, CSCW, Article 147 (Nov. 2019), 32 pages. https://doi.org/10.1145/3359249

[18] William F. Chaplin, Oliver P. John, and Lewis R. Goldberg. 1988. Conceptions of states and traits: dimensional attributes with ideals as prototypes. fournal of personality and social psychology 54, 4 (1988), 541.

[19] Hyunghoon Cho, Daphne Ippolito, and Yun William Yu. 2020. Contact tracing mobile apps for COVID-19: Privacy considerations and related trade-offs. arXiv:2003.11511.

[20] Scott Clifford, Ryan M. Jewell, and Philip D. Waggoner. 2015. Are samples drawn from Mechanical Turk valid for research on political ideology? Research \& Politics 2, 4 (2015), 1-9. https://doi.org/10.1177/2053168015622072

[21] Simon P. Cohn. 2006. Privacy and confidentiality in the nationwide health information network. http://www.ncvhs.hhs.gov/060622lt.htm

[22] Bipin C. Desai. 2020. Pandemic and Big Tech. In Proceedings of the 24th Symposium on International Database Engineering \& Applications (Seoul, Republic of Korea) (IDEAS '20). Association for Computing Machinery, New York, NY, USA, Article 20, 10 pages. https://doi.org/10.1145/3410566.3410585

[23] Carsten F. Dormann, Jane Elith, Sven Bacher, Carsten Buchmann, Gudrun Carl, Gabriel Carré, Jaime R. García Marquéz, Bernd Gruber, Bruno Lafourcade, Pedro J. Leitão, Tamara Münkemüller, Colin McClean, Patrick E. Osborne, Björn Reineking, Boris Schröder, Andrew K. Skidmore, Damaris Zurell, and Sven Lautenbach. 2013. Collinearity: a review of methods to deal with it and a simulation study evaluating their performance. Ecography 36, 1 (2013), 27-46. https://doi.org/10.1111/j.1600-0587.2012.07348.x

[24] Martin French and Torin Monahan. 2020. Dis-ease surveillance: How might surveillance studies address COVID-19? Surveillance \& Society 18, 1 (2020), 1-11.

[25] Bram M. Fridhandler. 1986. Conceptual note on state, trait, and the state-trait distinction. Fournal of Personality and Social Psychology 50, 1 (1986), 169.

[26] Huiqing Fu and Janne Lindqvist. 2014. General area or approximate location? How people understand location permissions. In Proceedings of the 13th Workshop on Privacy in the Electronic Society. Association for Computing Machinery, Scottsdale, AZ, 117-120.

[27] Radhika Garg and Jenna Kim. 2018. An exploratory study for understanding reasons of (not-)using Internet of Things. In Extended Abstracts of the $2018 \mathrm{CHI}$ Conference on Human Factors in Computing Systems (Montreal QC, Canada) (CHI EA '18). Association for Computing Machinery, New York, NY, USA, 1-6. https://doi.org/10.1145/3170427.3188466

[28] Jeremy Ginsberg, Matthew H. Mohebbi, Rajan S. Patel, Lynnette Brammer, Mark S. Smolinski, and Larry Brilliant. 2009. Detecting influenza epidemics using search engine query data. Nature 457, 7232 (2009), 1012-1014.

[29] Joseph K. Goodman, Cynthia E. Cryder, and Amar Cheema. 2013. Data collection in a flat world: The strengths and weaknesses of Mechanical Turk samples. fournal of Behavioral Decision Making 26, 3 (2013), 213-224.

[30] Kevin D. Haggerty and Richard V. Ericson. 2000. The surveillant assemblage. The British fournal of Sociology 51, 4 (2000), 605-622. https://doi.org/10.1080/ 00071310020015280 
[31] Eszter Hargittai, Minh Hao Nguyen, Jaelle Fuchs, Jonathan Gruber, Will Marler Amanda Hunsaker, and Gokce Karaoglu. 2020. COVID-19 study on digital media and the coronavirus pandemic. http://webuse.org/covid/

[32] Eszter Hargittai and Elissa M. Redmiles. 2020. Will Americans be willing to install COVID-19 tracking apps? https://blogs.scientificamerican.com/observations/ will-americans-be-willing-to-install-covid-19-tracking-apps/ Library Catalog blogs.scientificamerican.com.

[33] Vi Hart, Divya Siddarth, Bethan Cantrell, Lila Tretikov, Peter Eckersley, John Langford, Scott Leibrand, Sham Kakade, Dana Lewis, Stefano Tessaro, and Glen Weyl. 2020. Outpacing the virus: Digital response to containing the spread of COVID-19 while mitigating privacy risks. https://ethics.harvard.edu/outpacingvirus

[34] Roberto Hoyle, Luke Stark, Qatrunnada Ismail, David Crandall, Apu Kapadia, and Denise Anthony. 2020. Privacy norms and preferences for photos posted online. ACM Transactions on Computer-Human Interaction (TOCHI) 27, 4 (2020), $1-27$.

[35] Human Rights Watch. 2020. COVID-19 Apps Pose Serious Human Rights Risks. https://www.hrw.org/news/2020/05/13/covid-19-apps-pose-serioushuman-rights-risks

[36] Edith Jacobson. 1965. The Self and the Object World. Hogarth Press, London.

[37] Immanuel Kant. 1999. Critique of Pure Reason. Cambridge University Press, Cambridge, UK.

[38] Michael Klenk, Hein Duijf, and Christian Engels. 2020. Ethics of digital contact tracing and COVID-19: Who is (not) free to go? https://papers.ssrn.com/sol3/ papers.cfm?abstract_id $=3595394$

[39] Christiane Kuhn, Martin Beck, and Thorsten Strufe. 2020. COVID notions: Towards formal definitions-and documented understanding-of privacy goals and claimed protection in proximity-tracing services. arXiv:2004.07723.

[40] Roberta Lamb and Rob Kling. 2003. Reconceptualizing users as social actors in information systems research. MIS Quarterly 27, 2 (2003), 197-236. http: //www.jstor.org/stable/30036529

[41] Alexander D. Langmuir. 1971. Communicable disease surveillance: Evolution of the concept of surveillance in the United States. Proceedings of the Royal Society of Medicine 64, 6 (1971), 681-684.

[42] Bruno Latour. 2005. Reassembling the social: An introduction to actor-network theory. Oxford University Press, Oxford, UK.

[43] Bruno Latour. 2020. Imaginer les gestes-barrières contre le retour à la production d'avant-crise. https://oara.fr/sites/default/files/images/upload/bruno_latour_ imaginer_les_gestes_barrie_res_1.pdf

[44] Leslie Lenert and Brooke Yeager McSwain. 2020. Balancing health privacy, health information exchange, and research in the context of the COVID-19 pandemic. https://doi.org/10.1093/jamia/ocaa039

[45] Jialiu Lin, Shahriyar Amini, Jason I. Hong, Norman Sadeh, Janne Lindqvist, and Joy Zhang. 2012. Expectation and purpose: Understanding users' mental models of mobile app privacy through crowdsourcing. In Proceedings of the 2012 ACM Conference on Ubiquitous Computing (Pittsburgh, Pennsylvania) (UbiComp '12) Association for Computing Machinery, New York, NY, USA, 501-510. https: //doi.org/10.1145/2370216.2370290

[46] Chris Y. T. Ma, David K. Y. Yau, Nung Kwan Yip, and Nageswara S. V. Rao. 2010 Privacy vulnerability of published anonymous mobility traces. In Proceedings of the Sixteenth Annual International Conference on Mobile Computing and Networking (Chicago, Illinois, USA) (MobiCom '10). Association for Computing Machinery, New York, NY, USA, 185-196. https://doi.org/10.1145/1859995.1860017

[47] Sascha Meinert. 2014. Field manual: Scenario building. https://www.etui.org/ sites/default/files/2014_Scenario_Building_DEF.pdf

[48] Georgina R. Mellor, Christine S. M. Currie, and Elizabeth L. Corbett. 2011. Incorporating household structure into a discrete-event simulation model of tuberculosis and HIV. ACM Trans. Model. Comput. Simul. 21, 4, Article 26 (Sept. 2011), 17 pages. https://doi.org/10.1145/2000494.2000499

[49] Maria D. Molina. 2019. I am what you eat: Effects of social influence on meal selection online. In Extended Abstracts of the 2019 CHI Conference on Human Factors in Computing Systems (Glasgow, Scotland UK) (CHI EA '19). Association for Computing Machinery, New York, NY, USA, 1-6. https://doi.org/10.1145/ 3290607.3308451

[50] Abu Saleh Md Noman, Sanchari Das, and Sameer Patil. 2019. Techies against Facebook: Understanding negative sentiment toward Facebook via user generated content. In Proceedings of the 2019 CHI Conference on Human Factors in Computing Systems (Glasgow, Scotland UK) (CHI '19). Association for Computing Machinery, New York, NY, USA, 1-15. https://doi.org/10.1145/3290605.3300698

[51] Cameron D. Norman and Harvey A. Skinner. 2006. eHEALS: The eHealth Literacy Scale. Journal of Medical Internet Research 8, 4 (2006), e27. https://doi.org/10. 2196/jmir.8.4.e27

[52] Sangchul Park, Gina Jeehyun Choi, and Haksoo Ko. 2020. Information Technology-Based Tracing Strategy in Response to COVID-19 in South KoreaPrivacy Controversies. https://jamanetwork.com/journals/jama/article-abstract/ 2765252

[53] Sameer Patil, Greg Norcie, Apu Kapadia, and Adam J. Lee. 2012. Reasons, rewards, regrets: Privacy considerations in location sharing as an interactive practice. In
Proceedings of the Eighth Symposium on Usable Privacy and Security (Washington, D.C.) (SOUPS '12). Association for Computing Machinery, New York, NY, USA, 1-15. https://doi.org/10.1145/2335356.2335363

[54] Aarathi Prasad, Jacob Sorber, Timothy Stablein, Denise Anthony, and David Kotz. 2012. Understanding sharing preferences and behavior for mHealth devices. In Proceedings of the 2012 ACM Workshop on Privacy in the Electronic Society (Raleigh, North Carolina, USA) (WPES '12). Association for Computing Machinery, New York, NY, USA, 117-128. https://doi.org/10.1145/2381966.2381983

[55] Davy Preuveneers and Wouter Joosen. 2016. Privacy-enabled remote health monitoring applications for resource constrained wearable devices. In Proceedings of the 31st Annual ACM Symposium on Applied Computing (Pisa, Italy) (SAC '16). Association for Computing Machinery, New York, NY, USA, 119-124. https: //doi.org/10.1145/2851613.2851683

[56] Ramesh Raskar, Isabel Schunemann, Rachel Barbar, Kristen Vilcans, Jim Gray, Praneeth Vepakomma, Suraj Kapa, Andrea Nuzzo, Rajiv Gupta, Alex Berke, et al. 2020. Apps gone rogue: Maintaining personal privacy in an epidemic. arXiv:2003.08567.

[57] Elissa M. Redmiles. 2020. User concerns \& tradeoffs in technology-facilitated contact tracing. arXiv:2004.13219.

[58] Elissa M. Redmiles, Sean Kross, and Michelle L. Mazurek. 2019. How well do my results generalize? Comparing security and privacy survey results from mturk, web, and telephone samples. In 2019 IEEE Symposium on Security and Privacy (SP). IEEE, San Francisco, CA, 1326-1343

[59] Leonie Reichert, Samuel Brack, and Björn Scheuermann. 2020. Privacy-preserving contact tracing of COVID-19 patients. https://eprint.iacr.org/2020/375.pdf Cryptology ePrint Archive, Report 2020/375, 2020.

[60] Jean-Jacques Rousseau. 1998. The Social Contract. Wordsworth Editions Limited, London.

[61] Kim Bartel Sheehan. 2018. Crowdsourcing research: data collection with Amazon's Mechanical Turk. Communication Monographs 85, 1 (2018), 140-156.

[62] Alessio Signorini, Alberto Maria Segre, and Philip M. Polgreen. 2011. The Use of Twitter to track levels of disease activity and public concern in the U.S. during the Influenza A H1N1 pandemic. PLOS ONE 6, 5 (05 2011), 1-10. https://doi.org/ 10.1371/journal.pone.0019467

[63] Theodore M. Singelis, Harry C. Triandis, Dharm P. S. Bhawuk, and Michele J. Gelfand. 1995. Horizontal and vertical dimensions of individualism and collectivism: A theoretical and measurement refinement. Cross-Cultural Research 29, 3 (1995), 240-275. https://doi.org/10.1177/106939719502900302

[64] Daniel J. Solove. 2012. Introduction: Privacy self-management and the consent dilemma. Harv. L. Rev. 126 (2012), 1880

[65] Elizabeth Stowell, Mercedes C. Lyson, Herman Saksono, Reneé C. Wurth, Holly Jimison, Misha Pavel, and Andrea G. Parker. 2018. Designing and evaluating mHealth interventions for vulnerable populations: A systematic review. In Proceedings of the 2018 CHI Conference on Human Factors in Computing Systems (Montreal QC, Canada) (CHI '18). Association for Computing Machinery, New York, NY, USA, 1-17. https://doi.org/10.1145/3173574.3173589

[66] Cass R. Sunstein. 2015. Nudging and choice architecture: Ethical considerations. Yale fournal on Regulation 32, 2 (2015), 413-450. https://www.yalejreg.com/ print/the-ethics-of-nudging/

[67] Jennifer Fries Taylor, Jodie Ferguson, and Pamela Scholder Ellen. 2015. From trait to state: Understanding privacy concerns. Journal of Consumer Marketing 32, 2 (2015), 99-112.

[68] Stephen B. Thacker and Ruth L. Berkelman. 1988. Public health surveillance in the United States. Epidemiologic reviews 10, 1 (1988), 164-190.

[69] Milka Trajkova and Aqueasha Martin-Hammond. 2020. "Alexa is a toy": Exploring older adults' reasons for using, limiting, and abandoning Echo. In Proceedings of the 2020 CHI Conference on Human Factors in Computing Systems (Honolulu, HI, USA) (CHI '20). Association for Computing Machinery, New York, NY, USA, 1-13. https://doi.org/10.1145/3313831.3376760

[70] Harry C. Triandis. 1996. The psychological measurement of cultural syndromes. American psychologist 51, 4 (1996), 407.

[71] Harry C. Triandis. 2001. Individualism-collectivism and personality. fournal of personality 69, 6 (2001), 907-924.

[72] Harry C. Triandis. 2018. Individualism and collectivism. Routledge, New York.

[73] Harry C. Triandis and Michele C. Gelfand. 1998. Converging measurement of horizontal and vertical individualism and collectivism. fournal of Personality and Social Psychology 74, 1 (1998), 118-128.

[74] Amos Tversky and Daniel Kahneman. 1981. The framing of decisions and the psychology of choice. Science 211, 4481 (1981), 453-458. https://doi.org/10.1126/ science.7455683

[75] Kristina P. Vatcheva, MinJae Lee, Joseph B. McCormick, and Mohammad H. Rahbar. 2016. Multicollinearity in regression analyses conducted in epidemiologic studies. Epidemiology (Sunnyvale, Calif.) 6, 2 (2016), 2161-1165.

[76] Rick Wash. 2010. Folk models of home computer security. In Proceedings of the Sixth Symposium on Usable Privacy and Security (Redmond, Washington, USA) (SOUPS '10). Association for Computing Machinery, New York, NY, USA, Article 11, 16 pages. https://doi.org/10.1145/1837110.1837125 
[77] Lucretia Williams, Gillian R. Hayes, Yuqing Guo, Amir Rahmani, and Nikil Dutt. 2020. HCI and mHealth wearable tech: A multidisciplinary research challenge. In Extended Abstracts of the 2020 CHI Conference on Human Factors in Computing Systems (Honolulu, HI, USA) (CHI EA '20). Association for Computing Machinery, New York, NY, USA, 1-7. https://doi.org/10.1145/3334480.3375223

[78] Jacob O. Wobbrock and Matthew Kay. 2016. Nonparametric statistics in humancomputer interaction. Springer International Publishing, Cham, 135-170. https //doi.org/10.1007/978-3-319-26633-6 7

[79] Heng Xu, Sumeet Gupta, Mary Beth Rosson, and John M. Carroll. 2012. Measuring mobile users' concerns for information privacy. In International Conference on Information Systems, Vol. 3. Association for Information Systems, Orlando, FL, USA, 2278-2293.

[80] Tyler M. Yasaka, Brandon M. Lehrich, and Ronald Sahyouni. 2020. Peer-to-peer contact tracing: Development of a privacy-preserving smartphone app. $7 M I R$ mHealth and uHealth 8, 4 (2020), e18936.
[81] Baobao Zhang, Sarah Kreps, and Nina McMurry. 2020. Americans' perceptions of privacy and surveillance in the COVID-19 pandemic. https://osf.io/9wz3y

[82] Xing Zhang, Shan Liu, Xing Chen, Lin Wang, Baojun Gao, and Qing Zhu. 2018. Health information privacy concerns, antecedents, and information disclosure intention in online health communities. Information \& Management 55, 4 (2018), 482-493. https://doi.org/10.1016/j.im.2017.11.003

[83] Yulei Zhang, Yan Dang, Yi-Da Chen, Hsinchun Chen, Mark Thurmond, ChwanChuen King, Daniel Dajun Zeng, and Catherine A. Larson. 2008. BioPortal infectious disease informatics research: Disease surveillance and situational awareness. In Proceedings of the 2008 International Conference on Digital Government Research (dg.o '08). Digital Government Society of North America, Montreal, Canada, 393-394.

[84] Shoshana Zuboff. 2019. The Age of Surveillance Capitalism: The Fight for a Human Future at the New Frontier of Power. PublicAffairs, New York. 


\section{A QUESTIONNAIRE}

\section{A.1 Screening Questions}

Participants were first asked screening questions to ensure consent and diligent participation. If either of these questions were answered negatively, participants were not allowed to complete the study.

- I have read and understood the information presented here and meet the conditions of eligibility. I agree to participate in this study.

- I have read and understood the information presented here and DO NOT meet the conditions of eligibility. I DO NOT agree to participate in this study.

(1) Will you provide your best answers to each question in this study?

- Yes; I will provide my best answers.

- No; I will not provide my best answers.

- I cannot promise either way.

\section{A.2 Scenario Context}

Participants were then given the scenario context:

During an outbreak of a new and highly contagious disease, the World Health Organization (WHO) recommends the use of a smart thermometer app to help minimize the spread of the disease. Apart from recording temperature, the app allows users to input additional symptoms they might be experiencing. Based on the symptoms, users receive suggestions for actions they should take to protect themselves and others in their communities from the contagious disease. The app uses a combination of Bluetooth and location data to measure exposure to the disease in communities. The app allows the data to be accessed by authorities, doctors, and scientists so that it can be used to track the spread of the disease and enforce people's compliance with containment measures, such as quarantines.

They were then asked the following questions with the respective answer choices:

(1) Which organization is recommending the app?

- $\mathrm{CDC}$

- WHO

- EU

- UNESCO

- TSA

- Other. Please specify:

(2) The app is primarily associated with which device?

- Smart Thermometer

- Smart Speaker

- Smart Watch

- Smart TV

- Fitness Tracker

- Other. Please specify:

\section{A.3 Scenarios}

If they were not in the control group, participants were then presented with two scenarios. These scenarios were beneficially and intrusively framed versions individualist or collectivist scenarios. After each scenario, participants were asked to indicate their levels of agreement based on the following options: Strongly disagree, disagree, somewhat disagree, neither agree nor disagree, somewhat agree, agree, and strongly agree. If they were in the control group, they were not shown a scenario but still asked about their agreement level.

Scenario provided to the participant. See Section $B$ for the text of the scenarios used in the various study conditions.

- When using the app, I would provide honest and accurate information about my symptoms.

- I would take the infection-prevention measures recommended by the app.

- If possible, I would continue to monitor my health using this app after the outbreak has been controlled.

- Usage of this app should remain optional at all times.

- Usage of this app should be mandatory at all times.

- Using an app to monitor and control the spread of this outbreak is beneficial.

- I am concerned that the information I submit to the app could be misused.

- I am concerned that others can find private information about me from this app.

- I am concerned about providing personal information to the app because of what healthcare providers might do with it.

- I am concerned about providing personal information to the app because of what the government might do with it.

- I am concerned about providing personal information to this app because it could be used in a way I did not foresee.

- In general, it would be risky to give personal information to the app.

- Health organizations (e.g., emergency services, governments, WHO) should have control over who can get access to my personal information collected by this app.

- There would be high potential for privacy loss associated with giving personal information to this app.

- Personal information could be inappropriately used by this app.

- Providing this app with my personal information would involve many unexpected problems.

- I should have control over who can get access to my personal information collected by this app.

- The government should have control over who can get access to my personal information collected by this app.

- The technology company responsible for making the app should have control over who can get access to my personal information collected by this app.

- This scenario could happen in the town or city where I live.

Participants were then given the following open-ended question:

- Please provide more detail about your answers to the questions above.

\section{A.4 Use of Health Technology}

Participants were then asked if the following statements applied to them based on the following options: Yes, unsure, or no.

- I have used health-related websites in the past (e.g., WebMD).

- I have used telehealth services in the past (e.g., phone/video consultations with medical professionals). 
- I have used specialized smart devices to measure various indicators of my health and exercise (e.g., thermometers, blood sugar monitors, weight scale, blood pressure cuffs, etc.).

They were then shown the following statements (eHEALS [51] and asked to indicate their levels of agreement based on the following options: Strongly disagree, disagree, somewhat disagree, neither agree nor disagree, somewhat agree, agree, and strongly agree.

- I know how to use health-related technologies to answer my health questions.

- I know what health-related technologies are available to me.

- I know how to use the information from health-related technologies to help me.

- Among the options available, select somewhat agree to show that you are reading attentively.

- I have the skills I need to evaluate the information I receive from health-related technologies.

- I can tell high quality from low quality health information received through health-related technologies.

- I feel confident in using health-related technologies to make health decisions.

\section{A.5 Health-Related}

They were then shown the following statements (MUIPC [79] and asked to indicate their levels of agreement based on the following options: Strongly disagree, disagree, somewhat disagree, neither agree nor disagree, somewhat agree, agree, and strongly agree.

- I am concerned that mobile apps are collecting too much information about me.

- I am concerned that mobile apps may monitor my activities on my mobile device.

- I feel that as a result of using mobile apps, others know more about me than I am comfortable with.

- I believe that as a result of my using mobile apps, information about me that I consider private is now more readily available to others than I would want.

- I feel that as a result of my using mobile apps, information about me is out there that, if used, will invade my privacy.

- I am concerned that mobile apps may use my personal information for other purposes without notifying me or getting my authorization.

- When I give personal information to use mobile apps, I am concerned that apps may use my information for other purposes.

- I am concerned that mobile apps may share my personal information with other entities without getting my authorization.

\section{A.6 Individualism and Collectivism}

They were then shown the following statements(Social orientation [73]) and asked to indicate their levels of agreement based on the following options: Strongly disagree, disagree, somewhat disagree, neither agree nor disagree, somewhat agree, agree, and strongly agree.

- I'd rather depend on myself than others.

- I rely on myself most of the time and rarely rely on others.

- I often do my own thing.
- My personal identity, independent of others, is very important to me.

- It is important for me to do my job better than others.

- Winning is everything.

- Competition is the law of nature.

- When another person does better than I do, I get tense and upset.

- If an acquaintance gets a prize, I would feel proud. Among the options available, select somewhat disagree to show that you are reading attentively.

- The well-being of my acquaintances is important to me.

- To me, pleasure is spending time with others.

- I feel good when I cooperate with others. Parents and children must stay together as much as possible.

- It is my duty to take care of my family, even when I have to sacrifice what I want.

- Family members should stick together no matter what sacrifices are required.

- It is important to me that I respect the decision made by groups.

\section{A.7 Demographics}

The following questions were shown to participants regarding their demographics:

(1) What is your year of birth?

(2) What is your gender

- Male

- Female

- Non-binary

- Prefer to self-describe:

(3) How many years have you lived in the USA?

(4) In which state do you live?

(5) How would you describe the locality where you live?

- Rural

- Suburban

- Urban

(6) Please indicate the types of devices you regularly use. (Select all that apply).

- Desktop Computer

- Laptop Computer

- Tablet

- Smartphone

- Gaming Console (e.g., XBox, Nintendo, Playstation, etc.)

- e-Reader

- Smart Watch

- Smart TV

- Smart Speaker (e.g., Amazon Echo, etc.)

- Fitness Trackers (e.g., FitBit)

- Robot Vacuum Cleaners (e.g., Roomba)

- Other. Please specify:

(7) What is the highest level of education you have completed? (If currently enrolled, highest degree received.)

- Less than high school

- High school graduate

- High school diploma

- Vocational training 
- Some college

- College graduate (B.S., B.A., or other 4 year degree)

- Master's degree

- Doctoral degree

- Professional degree after college (e.g., law or medical school)

(8) What is your marital status?

- Married

- Widowed

- Divorced

- Separated

- Never married

(9) Do you have any children below the age of 18 ?

- Yes

- No

(10) What is your ethnic background?

- American Indian or Native American

- Asian

- Black or African American

- Native Hawaiian or Other Pacific Islander

- White

- Hispanic

- Latino/Latina

- Other. Please specify:

- Prefer not to say

(11) Generally speaking, do you consider yourself a Republican, a Democrat, or an Independent?

- Strongly Republican

- Weakly Republican

- Independent with a lean toward the Republican party

- Independent with a lean toward the Democratic party

- Weakly Democrat

- Strongly Democrat

- Other. Please specify:

- Prefer not to say

(12) What is your annual household income?

(13) What is your current employment status?

- Employed full-time

- Employed part-time

- Unemployed looking for work

- Unemployed not looking for work

- Homemaker

- Student

- Retired

- Disabled

- Other. Please specify:

- Prefer not to say

(14) If user selects current employment status as full-time or part-time, the following question is displayed: What is your current profession?
(15) If user selects current employment status as student, the following question is displayed: What is your field of study?

\section{A.8 Anything Else}

(1) Is there anything else you would like to tell us?

\section{B SCENARIOS}

All participants received the Control Group text, which provided context and general information about a smart thermometer app intended to track the spread of infection. All non-control participants were then presented with two individually (IND) or two collectively (COL) framed scenarios. Following presentation of these scenarios, participants were asked to answer a series of questions pertaining to them, as seen above. Participants subsequently completed additional measures, as described above.

- Control Group Text: During an outbreak of a new and highly contagious disease, the World Health Organization (WHO) recommends the use of a smart thermometer app to help minimize the spread of the disease. Apart from recording temperature, the app allows users to input additional symptoms they might be experiencing. Based on the symptoms, users receive suggestions for actions they should take to protect themselves and others in their communities from the contagious disease. The app uses a combination of Bluetooth and location data to measure exposure to the disease in communities.

The app allows the data to be accessed by authorities, doctors, and scientists so that it can be used to track the spread of the disease and enforce people's compliance with containment measures, such as quarantines.

- IND/BEN Text: A few days ago, you personally started using the WHO-recommended smart thermometer app. Ten days after starting to use the app, you have followed the app's recommended actions and remain uninfected.

- IND/INT Text: A few days ago, you personally started using the WHO-recommended smart thermometer app. Ten days after starting to use the app, local emergency services inform you that the app data has flagged you for a 14-day quarantine and health monitoring.

- COL/BEN Text: A few days ago, everyone in your community started using the WHO-recommended smart thermometer app. Ten days after starting to use the app, the community has followed the app's recommended actions and the community infection rate is negligible.

- COL/INT Text: A few days ago, everyone in your community started using the WHO-recommended smart thermometer app. Ten days after starting to use the app, local emergency services inform the community that the app data has flagged the community for a 14-day quarantine and health monitoring. 